\title{
Analysis of Fast Ionization Wave Discharge Propagation in a Rectangular Geometry
}

\author{
Keisuke Takashima ${ }^{1}$, Igor V. Adamovich ${ }^{2}$, Zhongmin Xiong ${ }^{3}$, Mark J. Kushner ${ }^{4}$, \\ Svetlana Starikovskaia ${ }^{5}$, Uwe Czarnetzki $^{6}$, and Dirk Luggenhölscher ${ }^{7}$
}

\begin{abstract}
Fast Ionization Wave (FIW), nanosecond pulse discharge propagation in nitrogen and helium in a rectangular geometry channel / waveguide is studied experimentally using calibrated capacitive probe measurements. The repetitive nanosecond pulse discharge in the channel was generated using a custom designed pulsed plasma generator (peak voltage 10-40 kV, pulse duration 30-100 nsec, voltage rise time $\sim 1 \mathrm{kV} / \mathrm{nsec}$ ), generating a sequence of alternating polarity high-voltage pulses at a pulse repetition rate of $20 \mathrm{~Hz}$. Both negative polarity and positive polarity ionization waves have been studied. Ionization wave speed, as well as time-resolved potential distributions and axial electric field distributions in the propagating discharge are inferred from the capacitive probe data. ICCD images show that at the present conditions the FIW discharge in helium is diffuse and volume-filling, while in nitrogen the discharge propagates along the walls of the channel. FIW discharge propagation has been analyzed numerically using quasi-one-dimensional and two-dimensional kinetic models in a hydrodynamic (drift-diffusion), local ionization approximation. The wave speed and the electric field distribution in the wave front predicted by the model are in good agreement with the experimental results. A self-similar analytic solution of the fast ionization wave propagation equations has also been obtained. The analytic model of the FIW discharge predicts key ionization wave parameters, such as wave speed, peak electric field in the front, potential difference across the wave, and electron density as functions of the waveform on the high voltage electrode, in good agreement with the numerical calculations and the experimental results.

${ }^{1}$ Post-Doctoral Researcher, Department of Mechanical and Aerospace Engineering, The Ohio State University, Columbus, OH 43021, USA

2 Professor, Department of Mechanical and Aerospace Engineering, The Ohio State University, Columbus, $\mathrm{OH}$ 43210, USA, Associate Fellow AIAA

${ }^{3}$ Research Scientist, Department of Electrical Engineering and Computer Science, University of Michigan, Ann Arbor, MI 48109, USA

${ }^{4}$ Director, Michigan Institute for Plasma Science and Engineering, George I. Haddad Professor of Engineering, Department of Electrical Engineering and Computer Science, University of Michigan, Ann Arbor, MI 48109, USA

${ }^{5}$ Directeur de Recherche, Ecole Polytechnique, Laboratory for Physics and Technology of Plasmas, 91128 Palaiseau Cedex, France

${ }^{6}$ Professor, Department of Physics and Astronomy, Ruhr University Bochum, 44780 Bochum, Germany

${ }^{7}$ Research Associate, Department of Physics and Astronomy, Ruhr University Bochum, 44780 Bochum, Germany
\end{abstract}




\section{Introduction}

Over the last three decades, Fast Ionization Wave (FIW) discharges have been extensively studied, both experimentally and theoretically. An overview of experimental results and theoretical models published before mid-1990's is given in review by Vasilyak et al. [1]. More recently, a significant amount of experimental data on FIW dynamics and structure has been obtained at Moscow Institute of Physics and Technology in 1994-2008 [2-8], in studies of fundamental kinetic processes in nanosecond pulse discharges and their applications. At the present time, there exists an extensive body of data on fast ionization wave speed and attenuation coefficient in various gases in a wide range of pressures and peak voltages, on wave front dynamics and mechanisms of its formation, and on generation of high-energy (runaway) electrons in the wave front. Recent experimental results also include sub-nanosecond wave front imaging [5], visible / UV emission spectra of FIW discharges [6,7], and inference of electric field and electron density distributions in the wave front from calibrated capacitive probe measurements [2].

The main difference between streamer discharges [9-12] and FIW discharges is that the latter demonstrate high spatial uniformity and reproducibility, thus allowing accurate measurements of discharge parameters in a repetitively pulsed mode. Similarity between these two types of discharges is due to the fact that key elementary processes controlling discharge propagation are electron impact ionization in the discharge front and photoionization. Due to the high reduced electric field in the discharge front [13], the role of high-energy electrons in ionization kinetics can be significant $[14,15]$. The transition between the streamer and the ionization wave mode is rather gradual. Thus, reducing the pressure while keeping the high voltage pulse waveform fixed increases the streamer diameter [12], and at sufficiently low pressures in a large-volume chamber the discharge transforms into a spherical wave propagating from the high-voltage electrode [16]. Previously, development and propagation of FIW discharges in long tubes (at the conditions when the electrode gap is much greater compared to the discharge tube diameter) has been studied at low pressures (1-10 Torr), at peak pulse voltages of $10-20 \mathrm{kV}$, and at relatively high pressures (up to $1 \mathrm{bar}$ ), at pulse voltages of $100-200 \mathrm{kV}$. In both cases, pulse voltage rise rate was of the order of $1 \mathrm{kV} / \mathrm{ns}$ or higher, and pulse duration was within a few tens of nanoseconds. It has been shown that at these conditions, the discharge always initiates near the high-voltage electrode, with the current loop closed by the displacement current, with the wave speed (determined from time-resolved measurements of electrical potential distributions and optical emission in the wave front) is in the range of $1-10 \mathrm{~cm} / \mathrm{hs}$. The dependence of the wave speed on pressure and discharge tube diameter is discussed in [17].

Current interest in characterization of FIW discharges is driven mainly by their potential for applications such as plasma chemical fuel reforming, plasma-assisted combustion, high-speed flow control, pumping of electric discharge excited lasers, and generation of high-energy electrons. A unique capability of FIW discharges to generate significant ionization and high concentrations of excited species at high pressures and over large distances, before ionization instabilities have time to develop, is very attractive for these applications. Recent advances in laser optical diagnostics, such as Thomson scattering, nanosecond and picosecond Coherent Anti-Stokes Raman Spectroscopy (CARS), Laser Induced Fluorescence (LIF) and Laser Collision Induced Fluorescence (LCIF) are finally making possible detailed, non-intrusive, spatially and time-resolved measurements of electron density and electric field distributions in 
high-speed ionization wave discharges, on nanosecond time scale. Insight into FIW formation and propagation dynamics also requires development of predictive kinetic models, and their validation by the new experimental results. Note that although numerical kinetic models may incorporate detailed kinetics of charged and neutral species in the propagating ionization wave front (including non-local electron kinetics), analytic models remain attractive due to their capability of elucidating fundamental trends of discharge development.

The main objectives of the present work are (i) development and testing of the experimental apparatus for studies of FIW discharges which lends itself to the use of advanced optical diagnostics, (ii) measurements of FIW speed and electric field in the wave front by a calibrated capacitive probe, (iii) comparison of the experimental results with kinetic modeling calculations, and (iv) development of a predictive analytic model of FIW propagation.

\section{Experimental}

The schematic of the experimental apparatus for studies of Fast Ionization Wave discharge propagation is shown in Fig. 1. The ionization wave is generated in a rectangular cross section quartz channel, between a high voltage electrode on the left and a grounded electrode on the right. The high voltage electrode is a $12 \mathrm{~mm}$ outside diameter, $10 \mathrm{~mm}$ inside diameter hollow cylinder $32.5 \mathrm{~mm}$ long, with a $10 \mathrm{~mm}$ thick circular base $45 \mathrm{~mm}$ in diameter, made of copper. The grounded electrode is a $16 \mathrm{~mm}$ thick, $100 \mathrm{~mm}$ x $100 \mathrm{~mm}$ square copper flange, with a circular port $25 \mathrm{~mm}$ in diameter. The $22 \mathrm{~mm} \times 13.5 \mathrm{~mm}$ outside dimension rectangular cross section, $20 \mathrm{~cm}$ long quartz channel with $1.75 \mathrm{~mm}$ thick walls is fused to two quartz tube sections $14 \mathrm{~cm}$ long and $25 \mathrm{~mm}$ in diameter on both ends, as shown in Fig. 1. The grounded flange is connected to two copper waveguide plates $76 \mathrm{~mm}$ wide, mounted above and below the quartz channel, as shown in Fig. 1. The distance between the waveguide plates can be adjusted. In the present experiments, the waveguide plates are set $40 \mathrm{~mm}$ apart. The distance between the edge of the high voltage electrode to the grounded electrode is $28 \mathrm{~cm}$. The high voltage electrode is placed between two Teflon flanges, as shown in Fig. 1, and is connected to a high voltage terminal of a custom-designed, high-voltage, nanosecond pulse generator. The grounded terminal of the pulse generator is connected to the waveguide plates, thus forming a plane geometry plasma waveguide. Both the Teflon flanges and the grounded copper flange are mounted on a base plate made of glass fiber-reinforced plastic.

In the present experiments, a capacitive probe has been used to detect the arrival of ionization wave generated when a voltage pulse is applied to the high voltage electrode and traveling along the waveguide. This technique has been extensively used previously for FIW discharge diagnostics [2-4]. The capacitive probe is mounted in a $5 \mathrm{~mm}$ wide slot machined in the top waveguide plate and tightened with set screws, with the probe tip extending into the waveguide through the slot. The probe can slide along the discharge channel, between the leftmost and the rightmost positions, $x=5 \mathrm{~cm}$ and $\mathrm{x}=25 \mathrm{~cm}$ from the edge of the high voltage electrode. Details of the probe operation and its use for the surface charge, electric potential, and electric field distribution measurements in nanosecond pulse discharges are discussed in [2]. The design of the probe used in the present experiments is described in greater detail in our recent publication [18]. Basically, the probe is a custom-made coaxial feed-through capacitor (a few $\mathrm{pF}$ ) connected to a Tektronix 3054B oscilloscope through a $50 \Omega$ terminator. The probe has a 
rectangular flat tip $3 \mathrm{~mm} \times 12 \mathrm{~mm}$, placed horizontally $8 \mathrm{~mm}$ above the top wall of the discharge channel. During the ionization wave propagation along the discharge channel, the probe operates as a capacitive voltage divider, together with a stray capacitance between the charged wall of the channel and the plasma formed by the ionization wave front. Basically, the probe detects surface charge buildup on the top wall of the channel. If the amplitude and the time response of the probe are known, time-resolved electric potential on the discharge channel wall at the location of the probe tip can be inferred from the probe signal. In the present work, time response and amplitude response of the probe are determined by calibration, as discussed in Section 3.

Optical access to the discharge channel, for discharge imaging and emission measurements, is available from the sides (see Fig. 1). Additional optical access is provided from the ends of the discharge channel, through the quartz windows fused to the ends of $25 \mathrm{~mm}$ diameter quartz endpieces at a Brewster angle and a hollow cylinder high-voltage electrode, as shown in Fig. 1. The endpieces are connected to $25 \mathrm{~mm}$ diameter, $25 \mathrm{~cm}$ long gas flow inlet and exhaust tubes, made of glass (see Fig. 1). The main purpose of this design is to have no grounded or floating metal parts close to the high voltage electrode, to preclude nanosecond pulse discharge propagation toward them. The present experiments have been conducted in nitrogen and in helium at pressures of $\mathrm{P}=10-20$ Torr. The baseline helium flow rate is $1 \mathrm{slm}$, which corresponds to the flow velocity through the discharge channel of approximately $\mathrm{u}=7 \mathrm{~m} / \mathrm{sec}$ at $\mathrm{P}=10$ torr. The baseline nitrogen flow rate is $100 \mathrm{sccm}(\mathrm{u}=0.7 \mathrm{~m} / \mathrm{sec}$ at $\mathrm{P}=10$ torr $)$. The flow rate through the discharge section and the pressure are controlled by mass flow controllers and by a shutoff valve between the test section and the vacuum pump.

The high-voltage nanosecond pulse generator used in the present experiments has been custom designed to generate alternating polarity pulses with peak voltage of $10-30 \mathrm{kV}$, pulse duration 30-60 nsec, and pulse repetition rate up to $50 \mathrm{kHz}$. In the present work, the pulser is operated at low repetition rates of 10-20 Hz. The high-voltage pulses are generated using magnetic pulse compression. The pulse output voltage is regulated by varying the voltage of a DC power supply (Heinzinger HN 1200-01, 0-1200 V, 0-100 mA) providing input to the pulse generator, $\mathrm{U}_{\mathrm{DC}}=500-800 \mathrm{~V}$. Increasing input $\mathrm{DC}$ voltage also reduces the output pulse duration. Figure 2 shows typical positive and negative polarity pulse shapes generated in nitrogen at $\mathrm{P}=10$ torr and $v=10 \mathrm{~Hz}$. The experimental voltage waveforms shown in Fig. 2 can be approximated by a Gaussian shape pulse,

$$
U=U_{\text {peak }} \exp \left[-\left(\frac{t_{0}-t}{\tau}\right)^{2}\right],
$$

with $\tau=45 \mathrm{nsec}, 38 \mathrm{nsec}, 30 \mathrm{nsec}$, and $26 \mathrm{nsec}$ for $\mathrm{U}_{\mathrm{DC}}=560 \mathrm{~V}, 640 \mathrm{~V}, 720 \mathrm{~V}$, and $800 \mathrm{~V}$, respectively (FWHM of $75 \mathrm{nsec}, 63 \mathrm{nsec}, 50 \mathrm{nsec}$, and $44 \mathrm{nsec}$ ). The high-voltage pulser also produces a trigger output pulse, generated $400 \mathrm{nsec}$ before the main high-voltage pulse, for synchronization with optical diagnostics. The jitter of the trigger pulse relative to the main pulse is low, about $1 \mathrm{nsec}$ for positive polarity pulses and less than $2 \mathrm{nsec}$ for negative polarity pulses.

\section{Kinetic models}

To model the ionization wave propagation in the rectangular geometry channel, shown schematically in Fig. 3 in a simplified quasi-two-dimensional geometry, we used a two-fluid 
hydrodynamic plasma model in the drift-diffusion approximation. In Fig. 3, the channel length and half-height are $L=30 \mathrm{~cm}$ and $a=0.5 \mathrm{~cm}$, respectively, the dielectric thickness is $h=1.5 \mathrm{~cm}$, and the dielectric constant is $\varepsilon=1$. The governing equations in the discharge channel are as follows,

$$
\begin{gathered}
\frac{\partial n_{+}}{\partial t}+\frac{d i v \vec{j}_{+}}{e}=v_{i} n_{e}-\beta n_{+} n_{e}, \quad \frac{\vec{j}_{+}}{e}=-D_{+} \nabla n_{+}+\mu_{+} n_{+} \vec{E} \\
\frac{\partial n_{e}}{\partial t}+\frac{d i v \vec{j}_{e}}{e}=v_{i} n_{e}-\beta n_{+} n_{e}, \quad \frac{\overrightarrow{j_{e}}}{e}=-D_{e} \nabla n_{e}-\mu_{e} n_{e} \vec{E} \\
\Delta \varphi=-\frac{e}{\varepsilon_{0}}\left(n_{+}-n_{e}\right), \quad \vec{E}=-\nabla \varphi
\end{gathered}
$$

where $\nabla=(\partial / \partial x, \partial / \partial y), \Delta=\nabla^{2}$. The potential distribution in the dielectric surrounding the plasma (see Fig. 3) is determined from the Laplace equation, $\Delta \varphi=0$. Boundary conditions on the positive polarity high-voltage electrode and on the grounded electrode are as follows,

$$
\begin{array}{cccc}
n_{i}(x=0)=0 & \partial n_{e} / \partial x(x=0)=0 & n_{e}(x=L)=0 & \partial n_{i} / \partial x(x=L)=0 \\
\varphi(x=0, t)=U(t) & \varphi(x=L, t)=0
\end{array}
$$

Initial conditions for the electron and ion densities and for the potential in the discharge channel are

$$
n_{e}=n_{+}=n_{o} \quad ; \varphi=0
$$

The surface charge density on the dielectric wall is

$$
\sigma_{w}=\varepsilon_{o}\left(\varepsilon E_{r w}^{+}-E_{r w}^{-}\right)
$$

where $E_{y w}^{+}$and $E_{y w}^{-}$are transverse electric fields on the wall (i.e. at $y=a$, see Fig. 3 ), on the plasma side and on the dielectric side, respectively.

Following the method developed by Lagarkov and Rutkevich [19], i.e. integrating Eqs. (2-4) over the transverse coordinate $(y)$, while assuming that the potential distribution has the same $y$-dependence as in a linear electrostatic wave and that transverse nonuniformity of the ionization wave is weak, Eqs. (2-4) can be reduced to a system with only one (axial) coordinate $x$,

$$
\begin{gathered}
\frac{\partial n_{+}}{\partial t}+\frac{\operatorname{div} \vec{j}_{+x}}{e}=v_{i} n_{e}-\beta n_{+} n_{e} \\
\frac{\partial n_{e}}{\partial t}+\frac{\operatorname{div} \vec{j}_{e x}}{e}-\mu_{e} n_{e} k^{2} \varphi=v_{i} n_{e}-\beta n_{+} n_{e} \\
\frac{\partial E_{x}}{\partial x}+k^{2} \varphi=\frac{e}{\varepsilon_{0}} \rho, \quad E_{x}=-\frac{\partial \varphi}{\partial x}
\end{gathered}
$$


In Eqs. $\left(2^{\prime}-4^{\prime}\right), \mathrm{k}$ is the wavenumber of a linear electrostatic wave formed in a weakly preionized, rectangular geometry waveguide (see Fig. 3) at the conditions when ionization is negligible [19],

$$
\tan (k a) \tan (k h)=\chi=\frac{\varepsilon}{1+\frac{\sigma}{\varepsilon_{0} f}}=\frac{\varepsilon}{1+\frac{e n_{e} \mu_{e} E}{\varepsilon_{0} f}}, \quad f=\frac{1}{\varphi} \frac{d \varphi}{d t}, \quad E \approx E_{x} .
$$

For small wavenumbers (i.e. long wavelengths), a simple asymptotic expression for the wavenumber is valid,

$$
k a<<1, \quad k h<<1: \quad k \approx\left(\frac{\chi}{a h}\right)^{1 / 2}
$$

In the present work, we assume that the rate of electron impact ionization is controlled by the local electric field. The expression for the ionization frequency, $v_{i}$, used in Eqs. $\left(2^{\prime}-4^{\prime}\right)$ is a fit to the experimental data on Townsend ionization coefficient in nitrogen and helium as a function of the reduced electric field, $E / p[20]$,

$$
\begin{aligned}
& \left(\frac{\alpha}{p}\right)_{N_{2}}=\left\{\begin{array}{l}
\frac{900}{E / p} \cdot \exp \left[-\frac{315}{E / p}\right] \mathrm{cm}^{-1}, \frac{E}{p}<100 \mathrm{~V} / \mathrm{cm} \cdot \text { torr } \\
12 \cdot \exp \left[-\frac{342}{E / p}\right] \mathrm{cm}^{-1}, 100<\frac{E}{p}<800 \mathrm{~V} / \mathrm{cm} \cdot \text { torr }
\end{array} \quad v_{i}=\alpha \mu_{e} E\right. \\
& \left(\frac{\alpha}{p}\right)_{\mathrm{He}}=3 \cdot \exp \left[-\frac{34}{E / p}\right] \mathrm{cm}^{-1}, 20<\frac{E}{p}<150 \mathrm{~V} / \mathrm{cm} \cdot \text { tor }
\end{aligned}
$$

This problem formulation also does not incorporate photo-ionization and ionization in collisions of excited species. A constant electron mobility, $\mu_{e} p=0.42 \cdot 10^{6} \mathrm{~cm}^{2} \cdot$ Torr $/ \mathrm{V} \cdot \mathrm{s}$ for $\mathrm{N}_{2}$ and $\mu_{e} p=0.86 \cdot 10^{6} \mathrm{~cm}^{2} \cdot$ Torr $/ \mathrm{V} \cdot \mathrm{s}$ for $\mathrm{He}$ [20], was assumed. Since both electron-ion recombination and ion drift are extremely slow on the time scale of wave propagation (tens of nanoseconds), the recombination rate coefficient, $\beta=10^{-8} \mathrm{~cm}^{3} / \mathrm{s}$, and the ion mobility, $\mu_{+} p=$ $0.21 \cdot 10^{4} \mathrm{~cm}^{2} \cdot$ Torr $/ \mathrm{V} \cdot \mathrm{s}$, have almost no effect on the results. Similarly, both electron diffusion and ion diffusion are completely negligible on this time scale. The initial electron density is taken to be $n_{0}=10^{7} \mathrm{~cm}^{-3}$.

In addition to 1-D simulations, two-dimensional simulations were performed with nonPDPSIM, a 2-D plasma hydrodynamics model with radiation photon transport. In nonPDPSIM, continuity equations for charged and neutral species, and Poisson's equation for electric potential are integrated coincidently in time with the electron energy equation, with transport coefficients obtained from stationary solutions of the Boltzmann equation. The use of the electron energy equation allows for non-equilibrium between the local electric field and electron transport coefficients. The spatial discretization in nonPDPSIM is based on a finite volume method on an unstructured mesh. The time integration of Poisson's equation and charged particle transport are implemented with a fully implicit Newton iteration method. The transport 
of photon radiation is addressed using a propagator - Green's function method. The details of the model and the solution method are described in Refs. [21,22].

The 2-D geometry used in the model is based on the experimental setup described above, shown in Fig. 1, including the powered and the grounded electrodes, the metal waveguide, and the dielectric discharge channel filled with nitrogen with a small amount of oxygen $\left(\mathrm{N}_{2} / \mathrm{O}_{2}=\right.$ $99.9 \% / 0.1 \%$ ) at 10 Torr and $300 \mathrm{~K}$. Adding a small amount of $\mathrm{O}_{2}$ does not significantly affect the FIW dynamics but does provide a mechanism for photo-ionization which, for a positive polarity discharge, is required to provide seed electrons ahead of the ionization front. The discharge is assumed to be symmetric about the channel center line. The initial electron density in the channel is assumed to be zero, except for a small spot of plasma $(0.5 \mathrm{~cm}$ in diameter, maximum electron density of $10^{10} \mathrm{~cm}^{-3}$ ) near the tip of the powered electrode. This seeding is required to initiate the plasma in the absence of electric field emission from the positively biased electrode. A nonzero electron density ahead of the ionization front, necessary for the propagation of the ionization front in the positive polarity case, is produced through photoionization of $\mathrm{O}_{2}$ additive by the UV photon flux from radiating nitrogen species $\mathrm{N}_{2}\left(\mathrm{C}^{3} \Pi_{\mathrm{u}}\right)$, generated behind the ionization front.

Both in the 1-D and in the 2-D modeling calculations, the experimental pulse voltage waveforms were approximated by a Gaussian shape pulse, given by Eq. (1).

\section{Results and discussion}

\subsection{Wave speed and electric field measurements}

In the present work, the capacitive probe was calibrated using two separate procedures. First, the time response of the probe was determined by placing the probe tip next to a coaxial cable transmitting a rectangular shape voltage pulse (peak voltage $4 \mathrm{~V}, 50$ nsec pulse duration, 8 nsec rise time, 7 nsec fall time) produced by a SRS DG535 delay generator. To detect the pulse, part of the cable coaxial shielding was removed. The response time of the probe, $\tau=8$ nsec, was found from the following equation [18],

$$
U(t)=A\left[V(t)+\frac{1}{\tau} \int_{0}^{t} V\left(t^{\prime}\right) d t^{\prime}\right],
$$

where $U(t)$ is the voltage pulse shape in the cable and $V(t)$ is probe signal. The amplitude response of the probe, $A$, was measured by placing a rectangular cross section shell made of copper foil inside the quartz discharge channel, with the foil following closely the channel walls. The shell was connected to the high-voltage electrode, powered by the high-voltage nanosecond pulse generator, and extended along the entire channel. Thus, a high-voltage pulse $U(t)$ of known amplitude and shape, measured on the electrode by a Tektronix P6015A high voltage probe, was applied to the entire shell. The amplitude response of the capacitive probe, with the tip located 8 $\mathrm{mm}$ above the quartz channel, was again obtained from Eq. (12), $A=5.0 \cdot 10^{3}$. After calibration, the height and the orientation of the probe tip were kept the same. As discussed in Section 2, for measurements at different locations the probe was translated along the discharge channel by sliding along the slot machined in the top waveguide plate. 
Figure 4(a) plots raw capacitive probe signals measured in helium, at $\mathrm{P}=20$ torr, $v=20 \mathrm{~Hz}$, and $\mathrm{U}_{\mathrm{DC}}=500 \mathrm{~V}$, for a positive polarity wave (pulse peak voltage $+10.5 \mathrm{kV}$ ). The probe signals are taken for multiple probe tip locations ranging from $x=5 \mathrm{~cm}$ to $\mathrm{x}=25 \mathrm{~cm}$ from the high voltage electrode, $1 \mathrm{~cm}$ apart. The traces in Fig. 4(a) are averaged over 200 positive polarity discharge pulses (i.e. 20 seconds). It can be seen that the results are reproduced very well run-to-run, with excellent signal-to-noise. Note that this is critical for inference of the electric field from the probe signals. The high-voltage pulse waveform is also shown in Fig. 4(a), on the same time scale. Figure 4(b) plots electric potentials at these probe locations, $\varphi(t)$, inferred from the raw probe signals, $V(t)$, using Eq. (12). The potentials in Fig. 4(b) are also plotted together with the high-voltage pulse (i.e. the potential on the high-voltage electrode, $U(t)$ ). Figure 4(b) illustrates that the wave of electric potential propagates to the right, from the high voltage electrode to the grounded electrode. The potentials measured by the probe start decreasing when the wave reaches the grounded electrode, which occurs approximately at $t=72 \mathrm{nsec}$. Figure 4(c) plots timeresolved axial electric field at midpoints between the probe locations, i.e. at $x=5.5,6.5, \ldots 24.5$ $\mathrm{cm}$, determined from the potential difference at these locations. It can be seen that the wave front initially steepens while peak electric field increases considerably (from less than $1 \mathrm{kV} / \mathrm{cm}$ to more than $2 \mathrm{kV} / \mathrm{cm}$ ), before reaching a quasi-steady-state shape (at $\mathrm{x} \approx 14 \mathrm{~cm}$ ), when peak electric field in the front starts gradually decreasing. Finally, Figure 4(d) plots the wave front location, as well as peak electric field and potential at the moment when the field peaks, vs. time. From Fig. 4(d), it can be seen that far from the high voltage electrode (at $x>11 \mathrm{~cm}$ ), the wave speed becomes nearly constant, $V=0.32 \mathrm{~cm} / \mathrm{nsec}$ at $\mathrm{x}=11-25 \mathrm{~cm}$. In this region, peak electric field in the wave front and the potential at the location of the peak field are gradually decaying, $\mathrm{E} \approx 2.0-1.8$ $\mathrm{kV} / \mathrm{cm}$ and $\mathrm{U}=5.0-4.0 \mathrm{kV}$, respectively.

Figures 5,6 plot time-resolved electric field and the wave trajectory, along with peak electric fields and corresponding potentials, for positive and negative polarity waves in nitrogen, at $\mathrm{P}=10$ torr, $v=20 \mathrm{~Hz}$, and $\mathrm{U}_{\mathrm{DC}}=640 \mathrm{~V}$ (pulse peak voltages +20 and $-21 \mathrm{kV}$, respectively). It can be seen that the negative polarity wave is more spread out, with peak electric field in the wave front not exceeding $\mathrm{E}=3 \mathrm{kV} / \mathrm{cm}$ (compared to approximately $\mathrm{E}=4 \mathrm{kV} / \mathrm{cm}$ in the positive polarity wave). In both cases, the wave initially steepens before reaching quasi-steady-state and decaying gradually. The negative polarity wave speed is significantly lower compared to that of the positive polarity wave, $V=0.53 \mathrm{~cm} / \mathrm{nsec}$ vs. $V=0.67 \mathrm{~cm} / \mathrm{nsec}$ (both values averaged over $\mathrm{x}=10-25$ $\mathrm{cm}$ ). The results obtained at a higher $\mathrm{DC}$ voltage, $\mathrm{U}_{\mathrm{DC}}=760 \mathrm{~V}$ (positive and negative pulse peak voltages of $+28.3 \mathrm{kV}$ and $-29.7 \mathrm{kV}$, respectively), are similar to the ones plotted in Figs. 5,6, and show similar trends. In this case, the wave speed was significantly higher, $V=1.02 \mathrm{~cm} / \mathrm{nsec}$ for positive polarity and $V=0.88 \mathrm{~cm} / \mathrm{nsec}$ for negative polarity (a difference of approximately $15 \%$ ), averaged over $\mathrm{x}=10-25 \mathrm{~cm}$.

Figure 7 shows broadband, 10-shot average ICCD images of positive and negative polarity ionization waves in helium, at $\mathrm{P}=20$ torr, $v=20 \mathrm{~Hz}$, and $\mathrm{U}=500 \mathrm{~V}$ (pulse peak voltages $+10.5 \mathrm{kV}$ and $-11.0 \mathrm{kV}$ ). In the images, the wave propagates left to right. The wave speed at these conditions is $V=0.32 \mathrm{~cm} / \mathrm{nsec}$ and $V=0.21 \mathrm{~cm} / \mathrm{nsec}$, respectively, such that the $4 \mathrm{nsec}$ camera gate provides spatial resolution of approximately $1 \mathrm{~cm}$. The field of view of the images is $4.6 \mathrm{~cm}$ by $3.1 \mathrm{~cm}$. The camera was triggered externally, by a trigger pulse provided by the highvoltage pulse generator. The trigger pulse jitter relative to the main high voltage pulse is low, about $1 \mathrm{nsec}$ for positive polarity pulses and less than $2 \mathrm{nsec}$ for negative polarity pulses. Pulse- 
to-pulse reproducibility of the ionization wave launch timing and wave speed and is so good that 10 -shot images shown appear essentially identical to the single-shot images. One can see that emission intensity distribution from the positive polarity wave is fairly uniform, while in the negative polarity wave emission is brighter near the top and bottom walls of the channel.

Figure 8 shows broadband single-shot ICCD images of positive and negative polarity ionization waves in nitrogen, at $\mathrm{P}=10$ torr, $v=20 \mathrm{~Hz}$, and $\mathrm{U}_{\mathrm{DC}}=500 \mathrm{~V}$ (pulse peak voltages +15.5 $\mathrm{kV}$ and $-16.0 \mathrm{kV}$ ). The wave speed at these conditions is $V=0.40 \mathrm{~cm} / \mathrm{nsec}$ and $V=0.36 \mathrm{~cm} / \mathrm{nsec}$, providing spatial resolution of approximately $1.5 \mathrm{~cm}$. From Figure 8 , it can be seen that the ionization wave in nitrogen propagates mainly along the walls of the discharge channel. This is consistent with the visual observation of the discharge through the windows in the endpieces of the discharge channel (see Fig. 1), which also shows that emission is brightest along the perimeter of the discharge channel.

\subsection{Comparison with numerical calculations}

Figure 9 compares the experimental results in helium, at $\mathrm{P}=20 \mathrm{torr}, v=20 \mathrm{~Hz}$, and $\mathrm{U}_{\mathrm{DC}}=500 \mathrm{~V}$, for a positive polarity wave with the modeling calculations using a quasi-onedimensional model described in Section 3 (pulse peak voltage $U_{\text {peak }}=+10.5 \mathrm{kV}, \tau=50 \mathrm{nsec}$ in Eq. (1)). The wave trajectory predicted by the model is in fairly good agreement with the experimental data. The predicted wave speed (i.e. the slope of the wave trajectory in Fig. 9) increases as the wave amplitude (peak electric field) is increased, and decreases when the wave begins to decay. Note, however, that the wave speed increase and reduction are not observed in the experiment, where the wave speed remains nearly constant both when its amplitude increases and when it begins to decay, $V=0.32 \mathrm{~cm} / \mathrm{nsec}$ at $\mathrm{x}=10-25 \mathrm{~cm}$ (see Fig. 4(d)). The model also considerably overpredicts peak electric field in the wave and potential at the peak field location in the region close to the high-voltage electrode, by more than a factor of two at $x=5-10 \mathrm{~cm}$.

Figure 10 compares the experimental results in nitrogen, at $\mathrm{P}=10$ torr, $v=20 \mathrm{~Hz}$, and $\mathrm{U}_{\mathrm{DC}}=760 \mathrm{~V}$ with the modeling calculations, for positive and negative polarity waves (pulse peak voltages of $U_{\text {peak }}=+28.3$ and $U_{\text {peak }}=-29.7 \mathrm{kV}, \tau=29 \mathrm{nsec}$ in Eq. (1)). In both these cases, the wave trajectory predicted by the model is in good agreement with the experimental data. The model predicts almost no difference in the wave speed for different polarity waves, although the average experimental speed of the positive polarity wave is approximately $15 \%$ higher compared to that of the negative polarity wave. Peak electric field in the positive polarity wave, predicted by the model, is also in good agreement with the experiment, although the potential at the peak field location is underpredicted, by $20-25 \%$ in the quasi-steady-state region. For the negative polarity wave, on the other hand, the predicted potential distribution is in good agreement with the data, while the field is underpredicted. The experimental data for the negative polarity wave have somewhat more scatter and not as well reproduced run-to-run, compared to the positive polarity data.

Figure 11 plots distributions of electron density, space charge density, $\rho=e\left(n_{+}-n_{-}\right)$, potential, and axial electric field in the FIW discharge in nitrogen at the conditions of Fig. 10, predicted by a quasi-one-dimensional FIW discharge model, for positive and negative polarity waves. All distributions are plotted for multiple moments of time $5 \mathrm{nsec}$ apart. Again, it can be seen that the model predicts almost no difference between the positive and the negative wave 
dynamics. As expected, the wave amplitude (peak electric field and peak charge density in the front) first increases with the potential on the high-voltage electrode, until wave gradual attenuation takes over at large distances from the electrode, and the amplitude is reduced. The predicted wave speed basically follows the amplitude, first increasing and then gradually decaying. The wave attenuation is caused by the residual electric field behind the wave, $\mathrm{E} \approx 1$ $\mathrm{kV} / \mathrm{cm}$, which results in a significant potential drop as the wave travels over a significant distance, $\Delta \mathrm{x}=10-20 \mathrm{~cm}$. At low pulse amplitudes and/or large distances between the electrodes, this effect may cause complete wave decay before it can reach the grounded electrode. The residual field behind the wave also results in additional ionization, thus coupling additional energy to the plasma and further increasing the electron density in the discharge channel. Note that since the wave speed appears to be controlled by the peak electric field in the front (i.e. by the potential difference across the wave), predicting it at long distances from the wave origin requires accurate prediction of the residual electric field behind the wave.

Figure 12 compares experimental and predicted time-resolved axial electric field in FIW discharge in nitrogen, at different distances from the high-voltage electrode at the conditions of Fig. 10(a) (for positive polarity wave). Note that for the purpose of comparing with the experimental data, the predicted electric field plotted in Fig. 12 has been approximated as a difference between the potentials at two axial locations $\Delta x=1 \mathrm{~cm}$ apart, i.e. $\mathrm{E}_{\mathrm{i}+1 / 2} \approx \varphi_{\mathrm{i}}-\varphi_{\mathrm{i}+1}$. This value is $15-20 \%$ lower than the actual electric field, $E_{x}=-d \varphi / d x$, calculated by the model. It can be seen that both peak electric field and the residual electric field in the plasma after the wave are predicted fairly accurately. Note that, compared to the experiment, the model predicts significantly higher peak electric field in the region near the high-voltage electrode (at $\mathrm{x}=6.5$ $10.5 \mathrm{~cm}$ ), where two-dimensional effects are expected to be most significant. Summarizing the results of Figs. 10-12, predictions of the quasi-one-dimensional numerical model of the FIW discharge in nitrogen are in fairly good agreement with experimental measurements of wave speed, peak electric field in the wave front, and the residual electric field behind the positive polarity wave. For the negative polarity wave, the wave speed predicted by the model matches the experimental results rather closely, although peak electric field in the front is significantly underpredicted. The level of agreement between the quasi-1-D model and the experiment is rather encouraging, considering significant transverse nonuniformity of FIW discharges in nitrogen, apparent from the images of Fig. 8.

Although the modeling calculations discussed above describe the majority of the experimentally observed trends, some aspects of the problem are not easily captured in the onedimensional model, such as radiation transport, charging of dielectric surfaces, and curvature of electric potential surfaces. To investigate the importance of these more subtle features, twodimensional modeling of the generation and propagation of a FIW in nitrogen was performed. A more detailed discussion of the multi-dimensional dynamics of FIW, with comparison with the present experiment, will be presented in a separate paper. Figure 13-15 show the results of 2-D modeling for the experimental conditions of Fig. 10 (a) (nitrogen, $\mathrm{P}=10$ torr, $v=20 \mathrm{~Hz}, \mathrm{U}_{\mathrm{DC}}=760$ $\mathrm{V}$, pulse peak voltage $U_{\text {peak }}=28.5 \mathrm{kV}$ achieved at $t_{0}=75 \mathrm{nsec}$, Gaussian pulse width parameter in Eq. (1) $\tau=29$ nsec).

In Figure 13, the electron temperature, $T_{e}$, the electron density $\mathrm{n}_{e}$, and the electric potential $\varphi$ are plotted at $\mathrm{t}=55,65$, and $75 \mathrm{~ns}$. The peak electron temperature is near $15 \mathrm{eV}$ and concentrated at the ionization front, where the electric field reaches values of $6.9 \mathrm{kV} / \mathrm{cm}$ 
(reduced electric field approximately $\mathrm{E} / \mathrm{N}=2100 \mathrm{Td}$ ). The potential drop across the ionization front varies in the range $10-12 \mathrm{kV}$. As the plasma column forms behind the ionization front having an electron density of $\mathrm{n}_{e} \sim 10^{12} \mathrm{~cm}^{-3}$, the anode potential is extended, resulting in a relatively low electric field $(600 \mathrm{~V} / \mathrm{cm}$, reduced electric field approximately $\mathrm{E} / \mathrm{N}=190 \mathrm{Td})$ in the bulk plasma. Using the electron temperature as a marker for the location of the ionization front, the speed of the ionization wave is found to be approximately $V=0.92 \mathrm{~cm} / \mathrm{nsec}$, slightly lower than in the experiment (see Fig. 10(a)). The electric potential contours show that the wall of the channel is rapidly charged by the passing ionization wave. These results also show that the structure of the ionization front does not significantly change as it propagates through the channel.

The electron density, the axial electric field, and the electron impact ionization source at the ionization front at $t=65 \mathrm{~ns}$ are plotted in Fig. 14. Peak values of these parameters, as well as of the photon production rate, are achieved close to the wall rather than near the channel center line. This is caused by the electric field enhancement near the wall due to the jump in permittivity, as well as by secondary photo-electron emission from the wall irradiated by the UV photons from the plasma. Qualitatively, peak electron production rate predicted near the wall is consistent with the experimental observations of optical emission in nitrogen (see Fig. 8). The peak electron density behind the ionization front, approximately $1.2 \cdot 10^{12} \mathrm{~cm}^{-3}$, is nearly independent of the axial location. The axial electric field, $\sim 6.9 \mathrm{kV} / \mathrm{cm}$, peaks in a narrow axial region approximately $5 \mathrm{~mm}$ thick and decays more rapidly behind the ionization front than ahead of it. The peak electron impact ionization rate is about $2.5 \cdot 10^{21} \mathrm{~cm}^{-3} \mathrm{~s}^{-1}$, achieved slightly behind the peak in the electric field, and emphasizes the short electron acceleration distance across the ionization front. Finally, the predicted time history of the axial electric field on the channel center line, for different axial locations of the capacitive probe, is shown in Fig. 15. For direct comparison with the experiments, the electric field here is obtained by using two-point potential difference, as was also done in the 1-D case (see Fig. 12). The resulting electric field is found to be $10 \%$ lower than the instantaneous local value produced by differentiation of the electric potential obtained from the model. The shape of the axial electric field is quite close to the experimental measurements, and the quantitative agreement at $\mathrm{x}>12.5 \mathrm{~cm}$ is rather good (compare with Fig. 12(a)). Near the powered electrode (at $x<12.5 \mathrm{~cm}$ ), the computed values are about a factor of two higher than in experiment (see Fig, 12(a)). However, this discrepancy is somewhat expected as the details of the 3-D, cylindrical shape powered electrode are not well captured in the 2-D planar simulations.

\subsection{Self-similar analytic solution for ionization wave propagation}

Following the approach [19] and introducing a self-similar spatial coordinate $\xi=x+V t$, where $\mathrm{V}$ is wave speed (such that $\partial / \partial x=\partial / \partial \xi, \partial / \partial t=V \partial / \partial \xi$ ), while neglecting ion drift, recombination, and diffusion on a short time scale, Eqs. $\left(2^{\prime}-4^{\prime}\right)$ can be reduced to a system of ordinary differential equations for the electron density (n), space charge density $(\rho)$ and axial electric field (E):

$$
\frac{d n}{d \xi}=\frac{v_{i} n}{V+w}
$$




$$
\begin{gathered}
\frac{d E}{d \xi}=k^{2} \varphi-\frac{e}{\varepsilon_{0}} \rho \\
\frac{d \rho}{d \xi}=\frac{\mu E v_{i} n}{V(V+w)}-\frac{e \mu n \rho}{\varepsilon_{0}(V+w)}
\end{gathered}
$$

Here, $w=\mu E$ is the electron drift velocity, such that $w<<V$. The difference between the positive and the negative polarity waves is that the sign of the drift velocity is changed from plus to minus. The ionization frequency is approximated as follows,

$$
v_{i}=A p \exp \left(-\frac{B p}{E}\right) \cdot \mu E
$$

where $A=12$ 1/cm·Torr and $B=342 \mathrm{~V} / \mathrm{cm} \cdot$ Torr for nitrogen (see Eq. (11)).

An approximate analytic solution of Eqs. (13-15) has been derived in Ref. [19], to predict the dependence of the ionization wave speed and amplitude (peak electric field) on the potential drop across the wave. However, an accurate self-similar solution for the electric field, electron density, and space charge density distributions in the wave front and behind the wave has not been obtained. Also, wave speed and amplitude have not been related to the voltage pulse parameters on the electrode (such as voltage rise time). In the present work, the self-similar solution is obtained in three separate regions, (I) "upstream of the wave" (before breakdown), (II) in the wave front (during breakdown), and (III) "downstream of the wave" (in the plasma shielded by space charge in the ionization wave front). In this solution, the wave speed, $V$, is assumed to be constant and is one of the input parameters of the model. A schematic of the quasi-one-dimensional ionization wave structure is shown in Fig. 16. The results for key parameter values and distributions in the wave are given below.

\section{Region I (linear wave before breakdown):}

Peak electric field in the ionization wave front, $E^{*}$, breakdown potential, $\varphi^{*}$, and the point where breakdown initiates, $\xi^{*}$, are given as follows:

$$
\begin{gathered}
v_{i}^{*} E^{*}=A p \exp \left(-\frac{B p}{E^{*}}\right) \cdot \mu E^{* 2}=2 B p V k_{0} \ln \left(\frac{k_{0} \varepsilon_{0} V}{e \mu n_{0}}\right), \quad \varphi^{*}=\frac{E^{*}}{k_{0}} \\
\xi^{*}=\frac{1}{k_{0}} \ln \left(\frac{E^{*}}{k_{0} \varphi_{0}}\right), \quad \eta^{*}=\xi-\xi^{*}
\end{gathered}
$$

Electron density and space charge density at breakdown point, $\eta^{*}$, are calculated as

$$
n^{*}=\frac{k_{0} \varepsilon_{0} V}{e \mu} \quad \rho^{*}=\frac{\mu E^{*} n^{*}}{V}=\frac{k_{0} \varepsilon_{0} E^{*}}{e}
$$

In Eqs. (17-19), $k_{0}$ is the wavenumber of a linear electrostatic wave "upstream" of breakdown point (at $\xi<\xi^{*}, \eta<0$ ), obtained from Eq. (9) when the plasma conductivity is very low, $\sigma<<\varepsilon_{0}(d \varphi / d t) / \varphi$, 


$$
\tan \left(k_{0} a\right) \tan \left(k_{0} h\right)=\varepsilon \quad k_{0} a<<1, \quad k_{0} h<<1: \quad k_{0} \approx\left(\frac{\varepsilon}{a h}\right)^{1 / 2}
$$

Electric field, potential, space charge, and electron density distributions in Region I are found from the following equations:

$$
\begin{aligned}
& E=E^{*} \exp \left(k_{0} \eta\right) \quad \varphi=\varphi^{*} \exp \left(k_{0} \eta\right) \quad \rho=\rho^{*} \exp \left(\frac{v_{i}^{*} \eta}{V}\right) \quad(\eta \leq 0) \\
& n=\left\{\begin{array}{cc}
n_{0}, & \eta \leq-\frac{E^{*}}{B p k_{0}} \\
n^{*} \exp \left[\frac{v_{i}^{*} \eta}{V}\left(1+\frac{B p k_{0}}{E^{*}} \frac{\eta}{2}\right)\right], & -\frac{E^{*}}{B p k_{0}} \leq \eta \leq 0
\end{array}\right.
\end{aligned}
$$

\section{Region II (primary breakdown wave):}

Electric field, potential, electron density, and space charge distributions in this region are calculated as follows,

$$
\begin{gathered}
E=E^{*} \exp \left[-\frac{v_{i}^{*} \eta}{V}\left(1-\exp \left(-\frac{B p}{E^{*}}\right)\right) \frac{E^{*}}{B p}\right] \\
\varphi=\varphi^{*}+\left\{1-\exp \left[-\frac{v_{i}^{*} \eta}{V}\left(1-\exp \left(-\frac{B p}{E^{*}}\right)\right) \frac{E^{*}}{B p}\right]\right\} \frac{B p V}{v_{i}^{*}\left(1-\exp \left(-\frac{B p}{E^{*}}\right)\right)} \\
n=n^{*}+\frac{\varepsilon_{0} v_{i}^{*}}{e \mu} \frac{E^{*}}{B p}\left[1-\exp \left(\frac{B p}{E^{*}} \frac{E-E^{*}}{E^{*}}\right)\right] \\
\rho=\left\{\begin{array}{l}
\frac{\mu E n}{V}, \\
\rho_{\text {peak }} \exp \left[-\frac{2 e \mu n_{\infty}}{\varepsilon_{0} V}\left(\eta-\eta_{\text {peak }}\right)\right], \quad \eta_{\text {peak }} \leq \eta
\end{array}\right.
\end{gathered}
$$

where

$$
\eta_{\text {peak }}=\frac{V B p}{2 v_{i}^{*} E^{*}} \frac{1}{\left[1-\exp \left(-\frac{B p}{E^{*}}\right)\right]}
$$

is the point where the space charge density peaks, with the peak value of 


$$
\rho_{\text {peak }}=\frac{\mu}{V} \frac{E^{*}}{\sqrt{e}}\left\{n^{*}+\frac{\varepsilon_{0} v_{i}^{*}}{e \mu} \frac{E^{*}}{B p}\left[1-\exp \left(-\frac{B p}{E^{*}} \frac{\sqrt{e}-1}{\sqrt{e}}\right)\right]\right\}
$$

Potential difference across the primary wave and the electron density after the primary wave can be found as

$$
\begin{gathered}
\varphi_{\infty}=\varphi^{*}+\frac{B p V}{v_{i}^{*}\left(1-\exp \left(-\frac{B p}{E^{*}}\right)\right)} \\
n_{\infty}=\frac{k_{0} \varepsilon_{0} V}{e \mu}+\frac{\varepsilon_{0} v_{i}^{*} E^{*}}{e \mu B p}\left[1-\exp \left(-\frac{B p}{E^{*}}\right)\right]=\frac{k_{0} \varepsilon_{0} V}{e \mu}\left\{1+2 \ln \left(\frac{k_{0} \varepsilon_{0} V}{e \mu n_{0}}\right)\left[1-\exp \left(-\frac{B p}{E^{*}}\right)\right]\right\}
\end{gathered}
$$

Region III (secondary linear wave and secondary ionization wave):

The boundary between regions II and III is found from the following matching condition,

$$
\rho=k_{\infty} \frac{\varepsilon_{0} E}{e}
$$

The wavenumber of the secondary linear wave, $k_{\infty}$, can be obtained from Eq. (9) when the plasma conductivity is high, $\sigma>>\varepsilon_{0}(d \varphi / d t) / \varphi$,

$$
k_{\infty}=\frac{\varepsilon \varepsilon_{0} V}{a h e \mu n_{\infty}}
$$

This gives the minimum value of the electric field downstream of the primary ionization wave, $E_{\min }$, and the location of the minimum, $\eta_{\min }$,

$$
E_{\min }=E^{*} \exp \left[-\frac{\alpha}{2(\alpha-\beta)}\right] \gamma^{-\frac{\beta}{\alpha-\beta}} \quad ; \quad \eta_{\min }=\frac{\alpha \eta_{\text {peak }}+\ln \gamma}{\alpha-\beta}
$$

where

$$
\alpha=\frac{2 e \mu n_{\infty}}{\varepsilon_{0} V} \quad \beta=\frac{v_{i}^{*}}{V}\left(1-\exp \left(-\frac{B p}{E^{*}}\right)\right) \frac{E^{*}}{B p} \quad \gamma=\frac{\rho_{\text {peak }} e}{\varepsilon_{0} k_{\infty} E^{*}}
$$

At $\eta=\eta_{\min }$, the electric field reaches minimum, $E=E_{\text {min }}$, since the field is shielded by the space charge in the primary wave front, and then starts increasing again, producing the secondary linear wave propagating over the plasma ionized by the primary ionization wave. When the field becomes sufficiently high for the ionization to begin again, the resultant charge separation causes the field to nearly level off, and it reaches a quasi-steady state value $E_{\max }$ near point $\eta_{\max }$, 


$$
\begin{gathered}
v_{i, \max }=\operatorname{Ap} \exp \left(-\frac{B p}{E_{\text {max }}}\right) \cdot \mu E_{\max }=k_{\infty} V \\
\eta_{\max }=\eta_{\text {min }}+\ln \left(\frac{E_{\text {max }}}{E_{\text {min }}}\right) \frac{1}{k_{\infty}}
\end{gathered}
$$

Ionization in this secondary wave starts affecting the electric field and the electron density distributions at the point $\eta_{\min }<\eta^{\prime}<\eta_{\max }$, such as

$$
\eta^{\prime}=\eta_{\min }+\ln \left(\frac{E^{\prime}}{E_{\min }}\right) \frac{1}{k_{\infty}}, \quad E^{\prime}=E_{\max }\left(1-\frac{E_{\max }}{B p}\right)
$$

Until this happens (i.e. at $\eta \leq \eta^{\prime}$ ), the field and the potential distributions both rise exponentially, while the electron density remains constant:

$$
\begin{gathered}
E=E_{\text {min }} \exp \left[k_{\infty}\left(\eta-\eta_{\text {min }}\right)\right] \\
\varphi=\varphi_{\infty}+\frac{E_{\text {min }}}{k_{\infty}}\left[\exp \left(k_{\infty}\left(\eta-\eta_{\min }\right)\right)-1\right] \\
n=n_{\infty}
\end{gathered}
$$

After ionization begins again (i.e. at $\eta^{\prime} \leq \eta$ ), the field, the potential, and the electron density are given as

$$
\begin{gathered}
E=E^{\prime} \frac{E_{\text {max }}}{E^{\prime}+\left(E_{\max }-E^{\prime}\right) \exp \left(-\frac{B p k_{\infty}\left(\eta-\eta^{\prime}\right)}{E_{\max }}\right)} \rightarrow E_{\max } \\
\varphi=\varphi_{\infty}+\frac{E^{\prime}-E_{\min }}{k_{\infty}}+E_{\max }\left\{\left(\eta-\eta^{\prime}\right)+\frac{E_{\max }}{B p k_{\infty}} \ln \left(\frac{E^{\prime}}{E}\right)\right\} \rightarrow \text { const }+E_{\text {max }}\left(\eta-\eta^{\prime}\right) \\
n=n_{\infty} \exp \left[k_{\infty}\left(\eta-\eta^{\prime}\right)\right] \frac{E^{\prime}}{E}
\end{gathered}
$$

At $\eta_{\max } \leq \eta$ the electron density continues to increase with distance approximately linearly,

$$
n=n_{\infty}\left[1+\frac{E_{\max }-E^{\prime}}{E^{\prime}}\left(\frac{E_{\max }}{E^{\prime}}\right)^{-B N / E_{\max }}\right]+n_{\infty} k_{\infty}\left(\eta-\eta_{\max }\right)=\operatorname{const}+\frac{\varepsilon \varepsilon_{0} V}{\text { ahe } \mu}\left(\eta-\eta_{\max }\right)
$$

Finally, space charge density in the entire Region III is calculated as 


$$
\rho=\frac{\varepsilon_{0} E v}{e V}
$$

The value of the "residual" electric field downstream of the wave, $E_{\max }$, given by Eq. (35), along with the rate of voltage rise on the high-voltage electrode, are the two critical parameters controlling the ionization wave speed and the wave attenuation coefficient. Indeed, since $E_{\text {max }}=d \varphi /\left.d \xi\right|_{\text {electrode }}=d U(t) / d \xi$, where $U(t)$ is the voltage on the electrode, and $V=d \xi / d t$, it is easy to see that to maintain constant wave speed, the voltage on the electrode needs to increase at the rate

$$
\frac{d U}{d t}=E_{\max } V
$$

On the other hand, if the voltage on the electrode, $U$, remains constant in time, the potential difference across the wave will decrease with distance, with the relative attenuation coefficient of

$$
\delta=\frac{1}{\varphi} \frac{d \varphi}{d \xi}=\frac{E_{\max }}{U}
$$

$\underline{\text { Breakdown field near electrode and initial wave speed: }}$

For a Gaussian high-voltage pulse, given by Eq. (1), breakdown field near the highvoltage electrode and the initial wave speed are given as follows,

$$
\begin{gathered}
v_{i, b r} \frac{E_{b r}}{B p}=2 f \ln \left(\frac{2 f \varepsilon_{0}}{e \mu n_{0}} \frac{1}{1+\frac{E_{b r}}{B p}}\right), \quad U_{b r}=\frac{E_{b r}}{k_{0}} \\
V_{b r}=\frac{2 f}{k_{0}} \frac{1}{1+\frac{E_{b r}}{B p}}
\end{gathered}
$$

where $\mathrm{U}_{\mathrm{br}}$ is breakdown voltage,

$$
U_{b r}=U_{p e a k} \exp \left[-\left(\frac{t_{0}-t_{b r}}{\tau}\right)^{2}\right] ; f=\frac{1}{U_{b r}} \frac{d U_{b r}}{d t}=\frac{2\left(t_{0}-t_{b r}\right)}{\tau^{2}}
$$

and $t_{b r}$ is breakdown time. Note, however, that Eqs. $(48,49)$ can be used only as estimates since in reality breakdown near the electrode may strongly depend on the electrode geometry and surface material.

Summarizing, key results characterizing fast ionization wave propagation include

(a) peak electric field in the primary ionization wave front, $E^{*}$, and potential difference across the primary wave, $\varphi^{*}$ ("breakdown potential" of the primary wave), Eq. (17) 
(b) electron density after the primary ionization wave, $n_{\infty}$, Eq. (30)

(c) quasi-steady-state "residual" electric field achieved in the secondary ionization wave, $E_{\max }$, Eq. (35)

(d) electron density rise rate in the secondary ionization wave, $d n / d \eta$, Eq. (44)

(e) voltage rise rate on the high-voltage electrode to maintain constant wave speed, $d U / d t$, Eq. (46)

(f) wave relative attenuation coefficient if the voltage on the electrode remains constant, $\delta$, Eq. (47)

To illustrate the accuracy of the present self-similar analytic solution, Fig. 17 compares a numerical solution of Eqs. $\left(2^{\prime}-4^{\prime}\right)$ and an analytic solution for the electron density, space charge density, potential, and axial electric field in the positive polarity ionization wave in nitrogen at $\mathrm{P}=10$ torr and $V=1 \mathrm{~cm} /$ nsec. This corresponds to the experimental conditions of Fig. 10(a), i.e. positive polarity wave in nitrogen, $\mathrm{P}=10$ torr, $\mathrm{v}=20 \mathrm{~Hz}, \mathrm{U}_{\mathrm{DC}}=760 \mathrm{~V}$, and nearly constant wave speed of $V=1.02 \mathrm{~cm} / \mathrm{nsec}$ at $\mathrm{x}=11-25 \mathrm{~cm}$. From Fig. 17, it can be seen that the analytic solution is in good agreement with the exact numerical solution. The model somewhat underestimates peak electric field, peak space charge density, and the potential difference across the primary wave. However, the shape of the electron density, electric field, and potential distributions are reproduced fairly well. In particular, the rate of electron density rise in the secondary ionization wave, as well as the residual electric field and the wave attenuation coefficient given by the numerical model and the analytic solution are close.

Figure 18(a) plots peak electric field in the wave front, $E^{*}$, and the residual electric field, $\underline{E}_{\max }$, vs. wave speed, $V$, for different initial electron densities in the channel. As expected, wave speed, which is controlled primarily by the rate of ionization in the wave front, $v_{i}$ (see Eq. (17)), has a strong (exponential) dependence on $E^{*}$. In other words, $E^{*}$ has logarithmic dependence on wave speed. Although peak electric field also has a relatively weak (double logarithmic) dependence on the initial electron density, $n_{0}$, it can be seen that increasing $n_{0}$ by several orders of magnitude reduces the peak field significantly. Finally, Eq. (17) shows that peak electric field in the wave front in fact depends on the product of the wave speed, $V$, and the wavenumber, $k_{0}$, which is controlled by the channel geometry and by the dielectric constant (see Eq. (20)). Therefore reducing the channel height, $2 a$, and the distance between the waveguide plates, $2 h$, as well as increasing the dielectric constant, $\varepsilon$, may substantially increase peak electric field in the wave. The residual electric field, $E_{\max }$, given by Eq. (35) also exhibits a weak logarithmic dependence on wave speed and is nearly unaffected by the initial electron density.

Figure $18(\mathrm{~b})$ shows that the electron density after the primary ionization wave, $n_{\infty}$, given by Eq. (30), increases with the wave speed almost linearly. In fact, it is proportional to the product $V k_{0}$, and therefore the electron density can also be increased by reducing the waveguide transverse dimensions and by using a dielectric with higher $\varepsilon$. At higher initial electron densities, $n_{\infty}$ is reduced significantly because charge separation in the wave front and self-shielding of the plasma occur more rapidly, thereby terminating ionization behind the wave front sooner.

Figure 18(c) demonstrates that maintaining higher wave speeds requires higher rate of voltage rise on the high-voltage electrode, $d U / d t$ (see Eq. (45)). Since the residual electric field, $E_{\text {max }}$, given by Eq. (35), exhibits relatively weak dependence on wave speed and initial electron 
density (see Fig. 18(a)), $d U / d t$ is the most critical parameter which controls wave speed. The present results predict that maintaining ionization wave speeds of $V=1-3 \mathrm{~cm} / \mathrm{nsec}$ in nitrogen requires rates of voltage increase of $d U / d t \sim 1-5 \mathrm{kV} / \mathrm{nsec}$. Finally, Fig. 18(d) plots estimated initial breakdown field and initial wave speed at its origin near the high-voltage electrode, predicted by Eqs. $(48,49)$, vs. voltage pulse width, $\tau$, for a Gaussian shape voltage pulse given by Eq. (1). It can be seen that a shorter voltage pulse (with a higher $d U / d t$ ) capable of maintaining a high-speed ionization wave also needs to have higher amplitude to generate breakdown near the electrode.

\section{Summary}

In the present work, Fast Ionization Wave discharges propagating along a rectangular geometry channel / plasma waveguide in nitrogen and helium are studied experimentally and using kinetic modeling. The repetitive nanosecond pulse discharge in the rectangular cross section channel was generated using a custom built pulsed plasma generator (peak voltage 10-40 $\mathrm{kV}$, pulse duration 30-100 nsec, voltage rise time $\sim 1 \mathrm{kV} / \mathrm{nsec}$ ), generating a sequence of alternating polarity high-voltage pulses at a pulse repetition rate of $20 \mathrm{~Hz}$. Using this plasma generator, both negative polarity and positive polarity ionization waves can be studied at the same experimental conditions, using a low-jitter output trigger produced by the generator on either positive or negative polarity pulses. The ionization wave speed, as well as time-resolved potential distributions and axial electric field distributions in the propagating FIW discharge have been inferred from the capacitive probe data. The probe is calibrated using voltage pulses of known pulse shape and amplitude.

The experiments have been conducted in helium and nitrogen, at pressures of 10-20 torr, at pulse peak voltages of $10-30 \mathrm{kV}$. As expected, wave speed increases with pulse peak voltage, as voltage rise time on the high-voltage electrode is reduced. Both the wave speed and the wave amplitude (peak axial electric field in the wave front) for the positive polarity wave exceed those for the negative polarity wave. ICCD camera images demonstrate that the ionization wave discharges in helium appear diffuse and volume-filling, although emission intensity in the negative polarity wave is higher near the top and bottom walls of the channel. On the other hand, ionization wave discharges in nitrogen propagate essentially along the walls of the discharge channel.

FIW discharge propagation has been analyzed numerically, using quasi-one-dimensional and two-dimensional kinetic models in a hydrodynamic (drift-diffusion), local ionization approximation. The wave speed and the electric field distribution in the wave front predicted by the model are in good agreement with the experimental results in nitrogen. A self-similar analytic solution of the fast ionization wave propagation equations has also been obtained. The analytic model of the FIW discharge predicts key ionization wave parameters, such as wave speed, peak electric field in the front, potential difference across the wave, and electron density as functions of the waveform on the high voltage electrode, in good agreement with the numerical calculations and the experimental results. 


\section{Acknowledgments}

This work has been supported by the U.S. Department of Energy Plasma Science Center, by the Department of Physics and Astronomy of the Ruhr-University Bochum, and by the Research Department "Plasmas with Complex Interactions" of the Ruhr-University Bochum. We would also like to thank Bernd Becker, Frank Kremer, Stefan Wietholt, and Thomas Zierow for their help with the experimental apparatus.

\section{References}

1. L.M. Vasilyak, S.V. Kostyuchenko, N.N. Kudryavtsev, and I.V. Filyugin, "Fast Ionization Waves Under Electrical Breakdown Conditions", Physics - Uspekhi 37 (1994) 247 - 269

2. N.B. Anikin, S.V. Pancheshnyi, S.M. Starikovskaia, and A.Yu. Starikovskii, "Breakdown development at high overvoltage: electric field, electronic levels excitation and electron density", J. Phys. D.: Appl. Phys. 31 (1998) 826-833

3. N.B. Anikin, S.M. Starikovskaia, and A.Yu. Starikovskii, "Uniform nanosecond gas breakdown of negative polarity: initiation form electrode and propagation in molecular gases", J. Phys. D: Appl. Phys. 34 (2001) 177-188

4. N.B. Anikin, S.M. Starikovskaia, and A.Yu. Starikovskii, "Polarity effect of applied pulse voltage on the development of uniform nanosecond gas breakdown", J. Phys. D: Appl. Phys. 35 (2002) 2785-2794

5. N.B. Anikin, N.A. Zavialova, S.M. Starikovskaia, and A.Yu. Starikovskii, "Nanosecond discharge development in long tubes", IEEE Trans. Plasma Sci. 36 (2008) 902-903

6. S.V. Pancheshnyi, S.M. Starikovskaia, and A.Yu. Starikovskii, "Population of nitrogen molecule electron states and structure of the fast ionization wave", J. Phys. D: Appl. Phys, 32 (1999) 2219-2227

7. S.V. Pancheshnyi, S.M. Starikovskaia, and A.Yu. Starikovskii, "Excitation of molecular hydrogen in the fast ionization wave", Plasma Physics Reports 25 (1999) 393-397

8. S.M. Starikovskaia, N.B. Anikin, S.V. Pancheshnyi, D.V. Zatsepin, and A.Yu. Starikovskii, "Pulsed breakdown at high overvoltage: development, propagation and energy branching", Plasma Sources Sci. Technol. 10 (2001) 344-356

9. I. Gallimberti, “A computer model for streamer propagation”, J. Phys. D: Appl. Phys. 5, (1972) 2179-2189

10. N.Yu. Babaeva and G.V. Naidis, "Simulation of positive streamers in air in weak uniform electric fields", Phys. Lett. A. 215 (1996) 187-190

11. E.M. van Veldhuizen and W.R. Rutgers, "Pulsed positive corona streamer propagation and branching”, J. Phys. D: Appl. Phys., 35 (2002) 2169-2179

12. S. Pancheshnyi, M. Nudnova, and A. Starikovskii, "Development of a cathode-directed streamer discharge in air at different pressures: experiment and comparison with direct numerical simulation", Phys. Rev. E, 71 (2005) 016407

13. S.V. Pancheshnyi, S.M. Starikovskaia, and A.Yu. Starikovskii, "Population of nitrogen molecule electron states and structure of the fast ionization wave", J.Phys.D: Appl.Phys. 32 (1999) 2219-2227

14. L.D. Tsendin, "Principles of the Electron Kinetics in Glow Discharges", in Electron Kinetics and Applications of Glow Discharges (U. Kortshagen, L.D. Tsendin, eds.), NATO ASI Series, Series B: Physics, vol. 367, pp. 1-18, Kluwer, New York, 1998 
15. E. Dewald, M. Gansiu, N.B. Mandache, G. Musa, M. Nistor, A.M. Pointu, I.-I. Popescu, K. Frank, D.H.H. Hoffmann, and R. Stark, "The role of multielectrode geometry in generation of pulsed intense electron beams in preionization controlled open ended hollow cathode transient discharges", IEEE Trans. Plasma Sci., 25 (1997) 279

16. S.M. Starikovskaia, A.Yu. Starikovskii, and D.V. Zatsepin, "Development of a spatially uniform fast ionization wave in a large discharges volume”, J. Phys. D: Appl. Phys. 31 (1998) 1118

17. E.I. Asinovsky, A.N. Lagarkov, V.V. Markovets, and I.M. Rutkevich, "On the similarity of electric breakdown waves propagating in shielded discharge tubes", Plasma Sources Sci. Technol., 3 (1994) 556

18. M. Nishihara, K. Udagawa Takashima, J.R. Bruzzese, I.V. Adamovich, and D. Gaitonde, "Experimental and Computational Studies of Low-Temperature Mach 4 Flow Control by Lorentz Force", J. Propulsion and Power, 27 (2011) 467-476

19. A.N. Lagarkov, I.M. Rutkevich, Ionization Waves in Electric Breakdown of Gases (Springer, New York, 1994), Chapter 4

20. Yu. P. Raizer, Gas Discharge Physics (Springer, Berlin, 1991), Chapters 2,4

21. M. J. Kushner, "Modeling of microdischarge devices: pyramidal structures", J. Appl. Phys. 95 (2004) 846

22. Z. Xiong and M. J. Kushner, "Surface corona-bar discharges for production of pre-ionizing UV light for pulsed high pressure plasmas", J. Phys. D: Appl. Phys. 43 (2010) 505204 


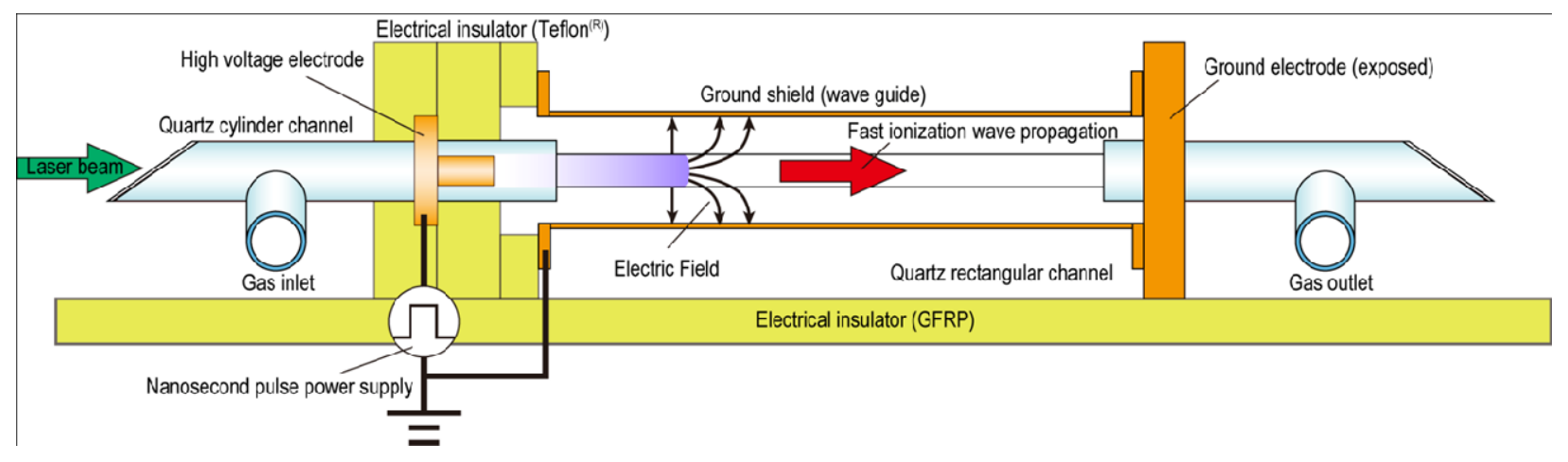

Figure 1. Schematic of the fast ionization wave discharge apparatus.

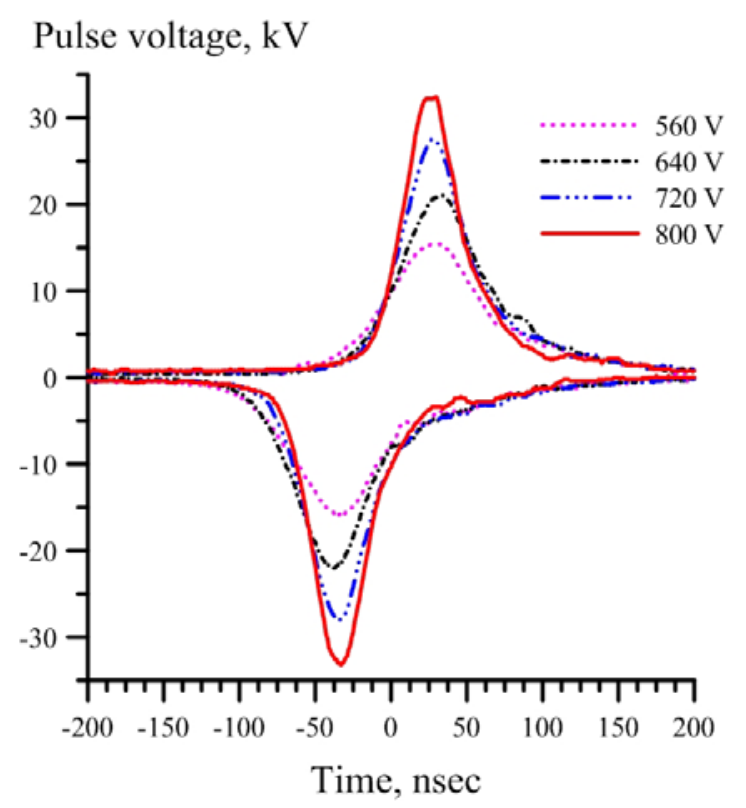

Figure 2. Typical positive and negative polarity voltage pulse waveforms generated by the pulsed plasma generator for different input DC voltages. 


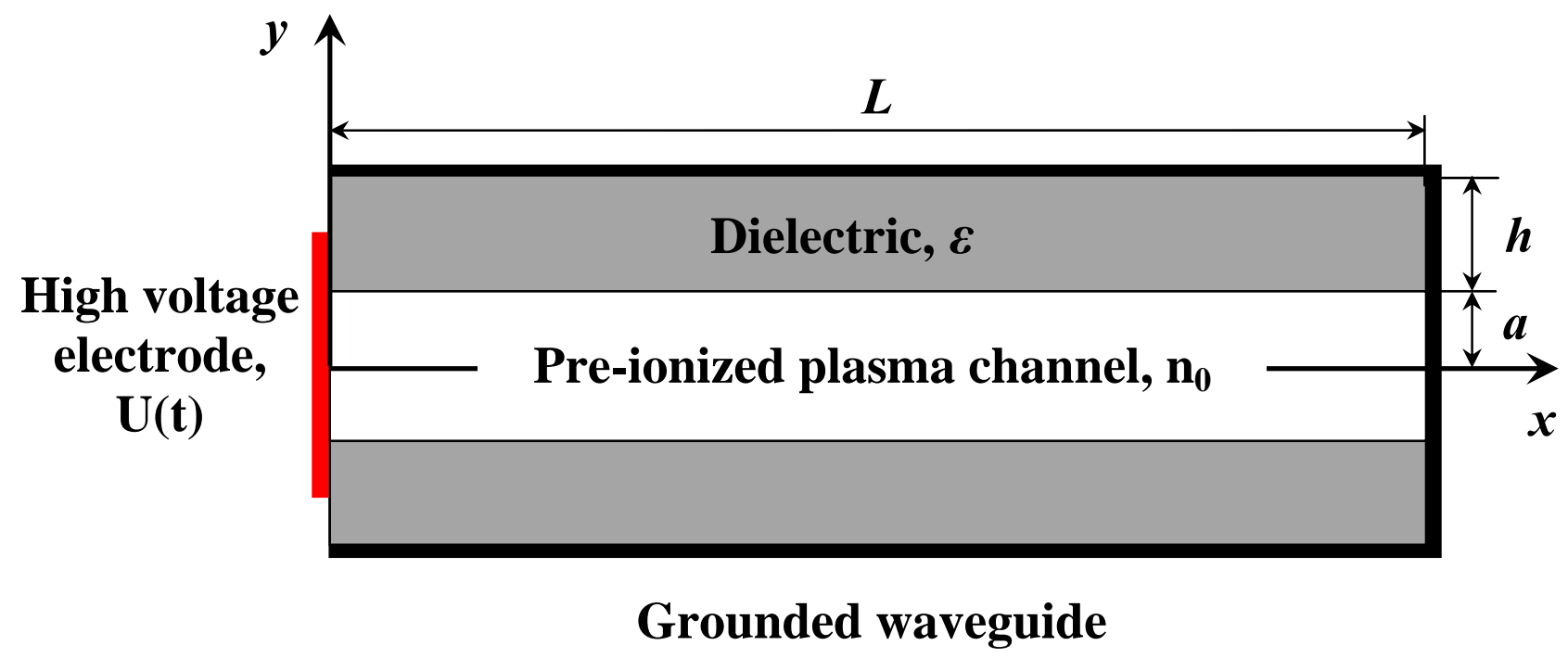

Figure 3. Schematic of the plasma channel and the dielectric layer in simplified quasi-two-dimensional geometry. 


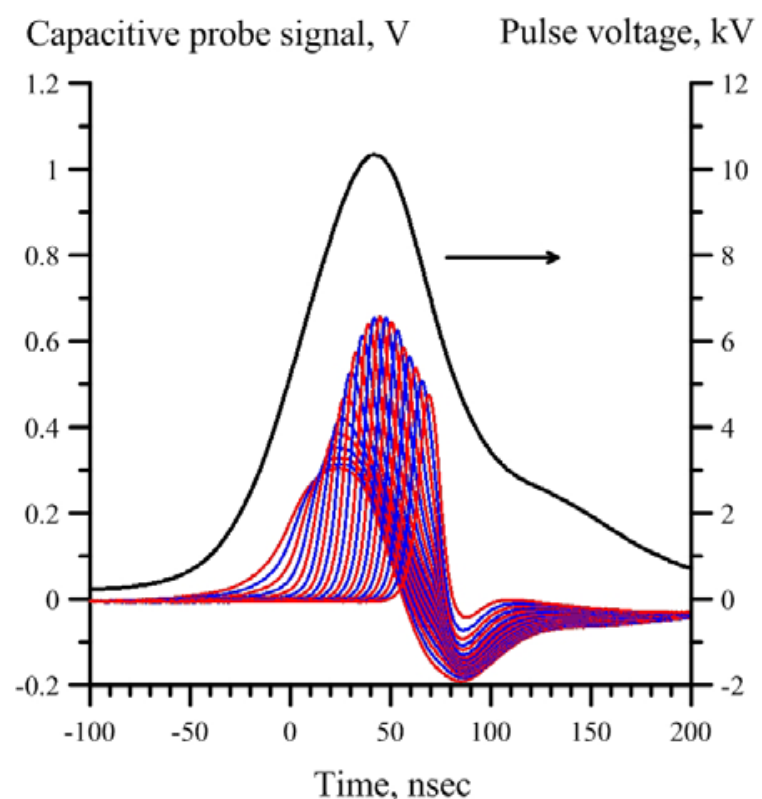

Time, nsec
Potential, V

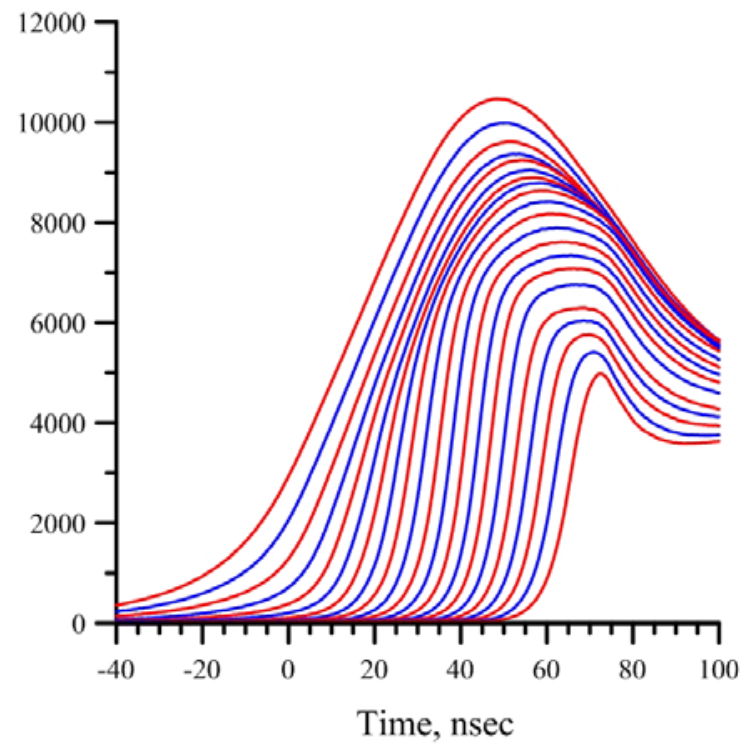

Potential, $\mathrm{kV}$

Distance, $\mathrm{cm}$

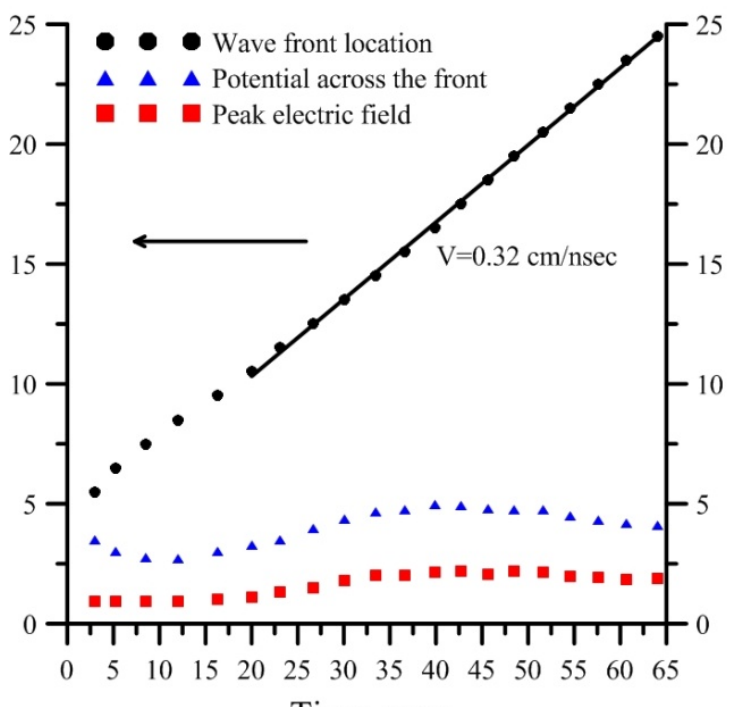

Time, nsec

Figure 4. (a) Raw capacitive probe data plotted together with the high voltage pulse waveform; (b) time-resolved potentials; (c) time-resolved axial electric fields; (d) ionization wave front location vs. time, peak electric field, and potential across the wave front at different probe locations. Data obtained for 21 different locations of the capacitive probe $1 \mathrm{~cm}$ apart, ranging from $5 \mathrm{~cm}$ to $25 \mathrm{~cm}$ from the high voltage electrode. Helium, $\mathrm{P}=20$ torr, $v=20 \mathrm{~Hz}, \mathrm{U}=500 \mathrm{~V}$, positive polarity wave (pulse peak voltage $+10.5 \mathrm{kV}$ ). 
Axial electric field, $\mathrm{V} / \mathrm{cm}$

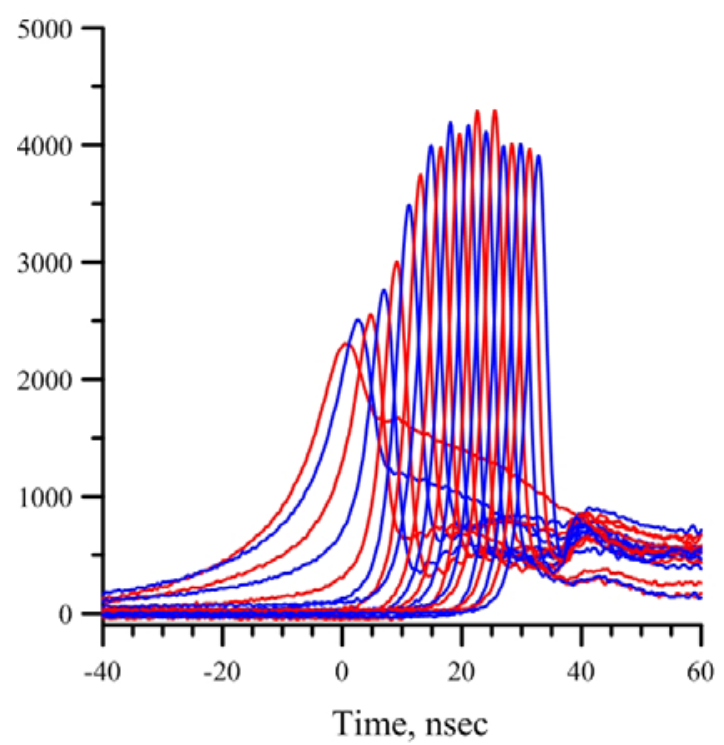

Distance, $\mathrm{cm}$

Potential, $\mathrm{kV}$

Electric field, $\mathrm{kV} / \mathrm{cm}$

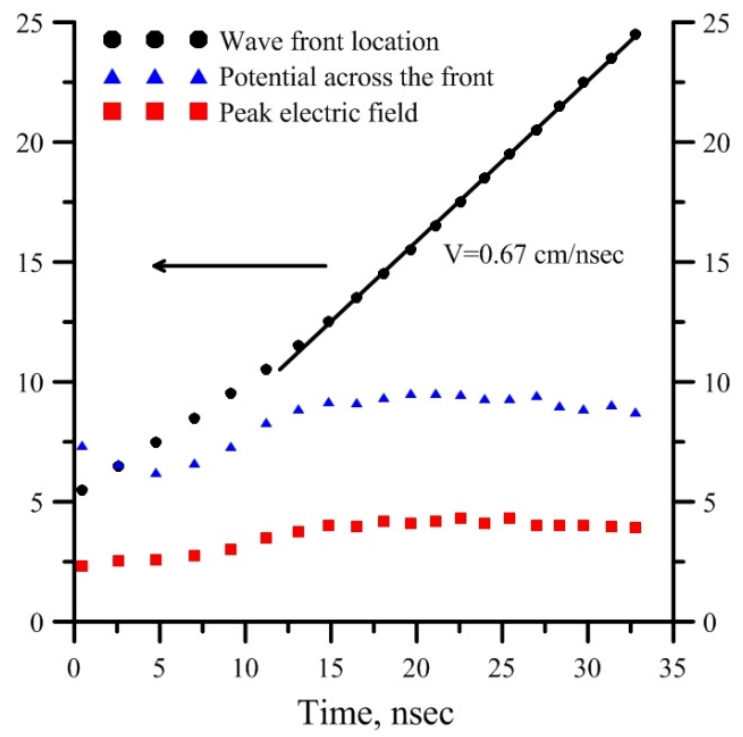

Figure 5. (a) Time-resolved axial electric fields; (b) ionization wave front location vs. time, peak electric field, and potential across the wave front at different probe locations. Nitrogen, $\mathrm{P}=10$ torr, $v=20 \mathrm{~Hz}, \mathrm{U}=640 \mathrm{~V}$, positive polarity wave (pulse peak voltage $+20 \mathrm{kV}$ ).

Axial electric field, $\mathrm{V} / \mathrm{cm}$

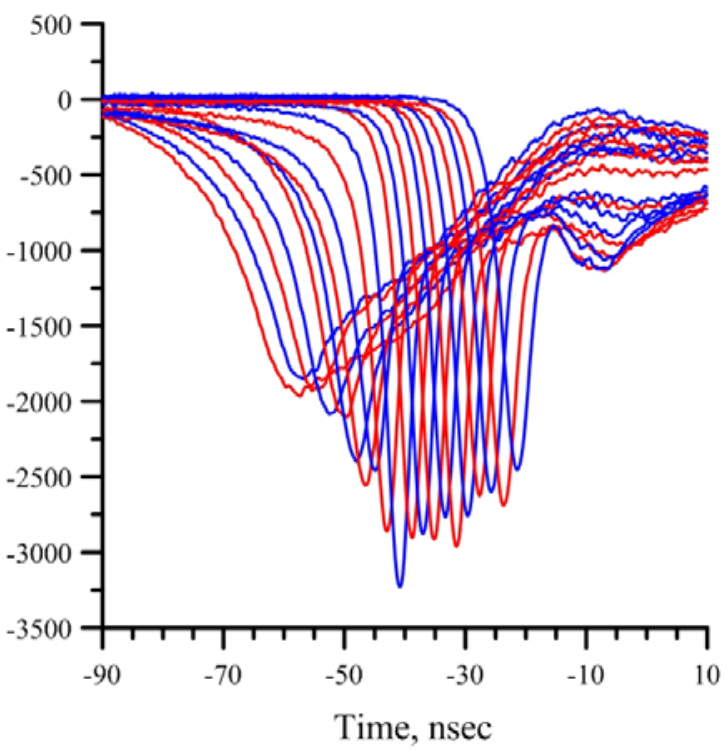

Potential, $\mathrm{kV}$

Electric field, $\mathrm{kV} / \mathrm{cm}$

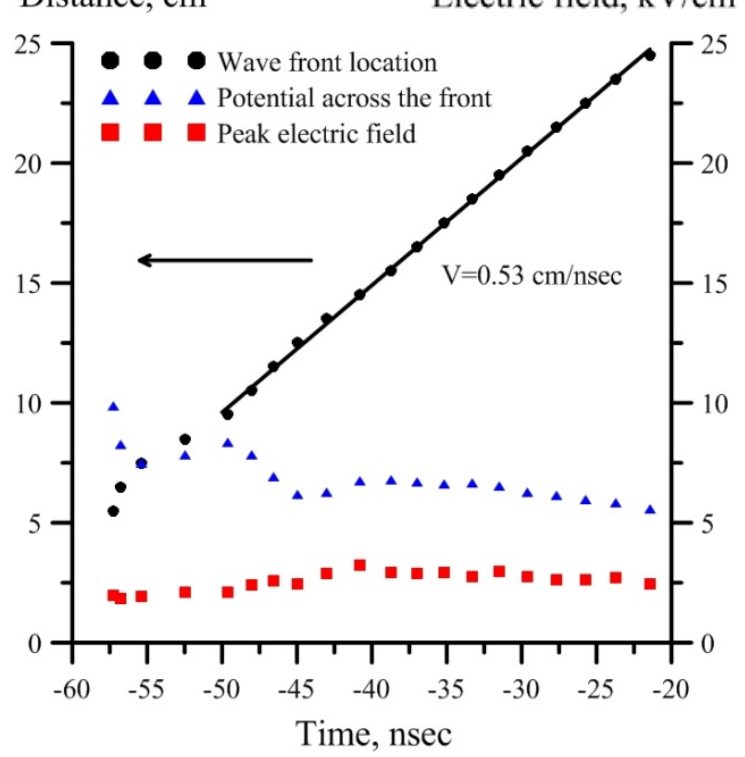

Figure 6. (a) Time-resolved axial electric fields; (b) ionization wave front location vs. time, peak electric field, and potential across the wave front at different probe locations. Nitrogen, $\mathrm{P}=10$ torr, $\mathrm{v}=20 \mathrm{~Hz}, \mathrm{U}=640 \mathrm{~V}$, negative polarity wave (pulse peak voltage $-21 \mathrm{kV}$ ). 


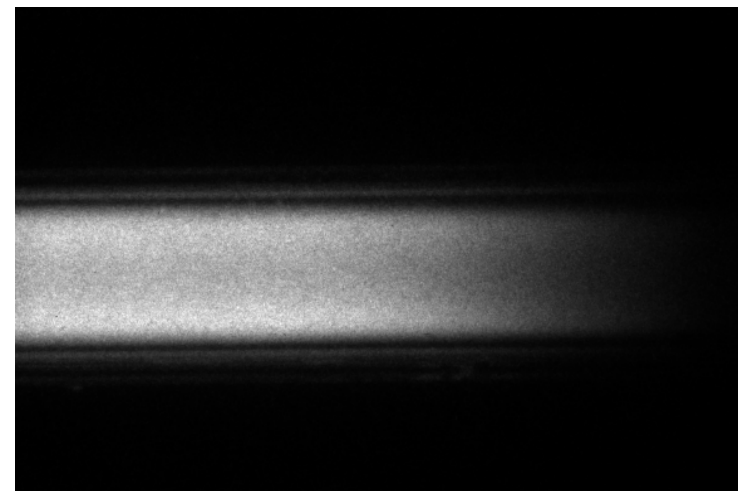

(a)

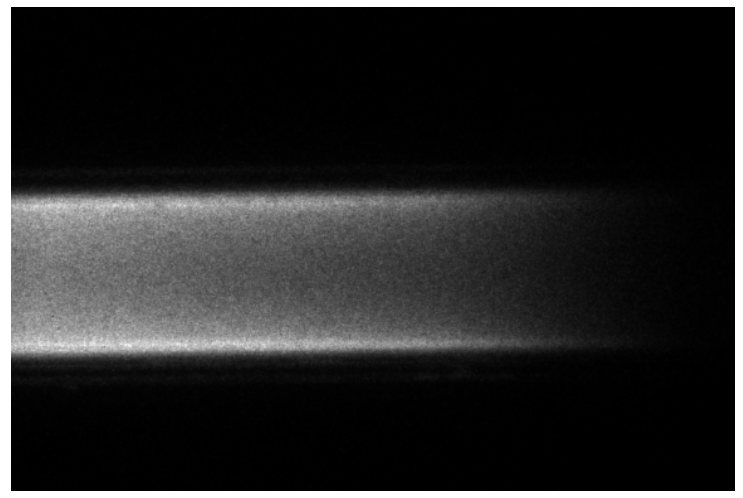

(b)

Figure 7. Broadband ICCD images (10-shot average) of (a) positive and (b) negative polarity ionization waves in helium, at $\mathrm{P}=20$ torr, $v=20 \mathrm{~Hz}$, and $\mathrm{U}=500 \mathrm{~V}$ (pulse peak voltages +10.5 $\mathrm{kV}$ and $-11.0 \mathrm{kV}$, respectively). Wave propagates left to right. Camera gate $4 \mathrm{nsec}$, providing spatial resolution of approximately $1 \mathrm{~cm}$. Field of view is $4.6 \mathrm{~cm}$ by $3.1 \mathrm{~cm}$.

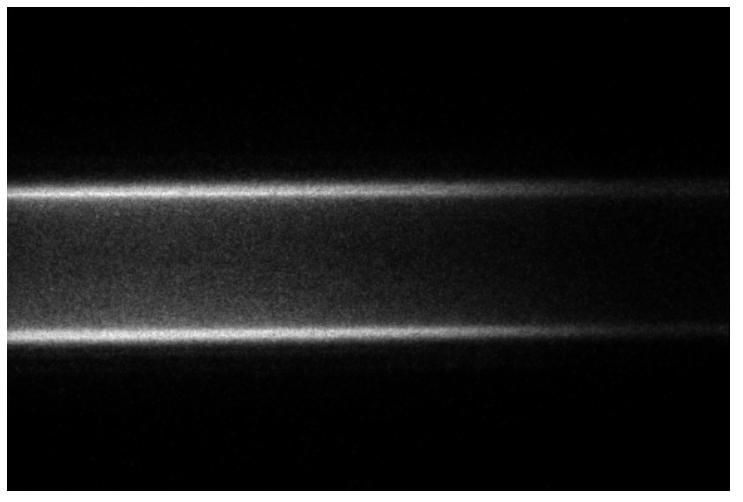

(a)

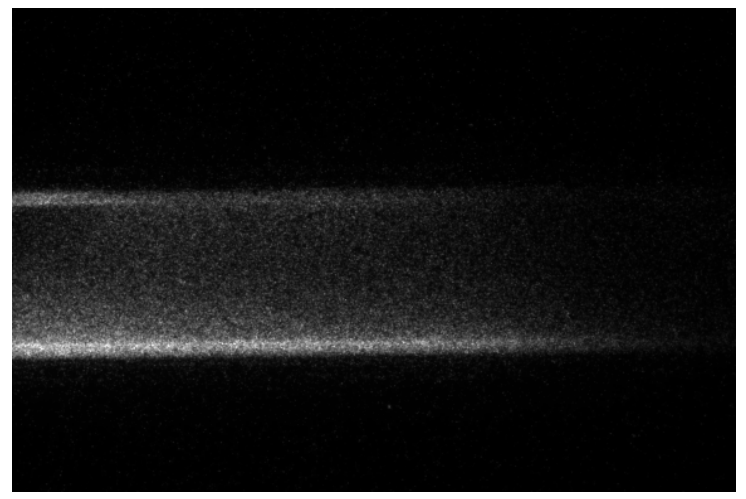

(b)

Figure 8. Broadband single-shot ICCD images of (a) positive and (b) negative polarity ionization waves in nitrogen, at $\mathrm{P}=10$ torr, $v=20 \mathrm{~Hz}$, and $\mathrm{U}=500 \mathrm{~V}$ (pulse peak voltages +15.5 $\mathrm{kV}$ and $-16.0 \mathrm{kV}$, respectively). Wave propagates left to right. Camera gate $4 \mathrm{nsec}$, providing spatial resolution of approximately $1.5 \mathrm{~cm}$. Field of view is $4.6 \mathrm{~cm}$ by $3.1 \mathrm{~cm}$. 
Potential, kV

Distance, $\mathrm{cm} \quad$ Electric field, $\mathrm{kV} / \mathrm{cm}$

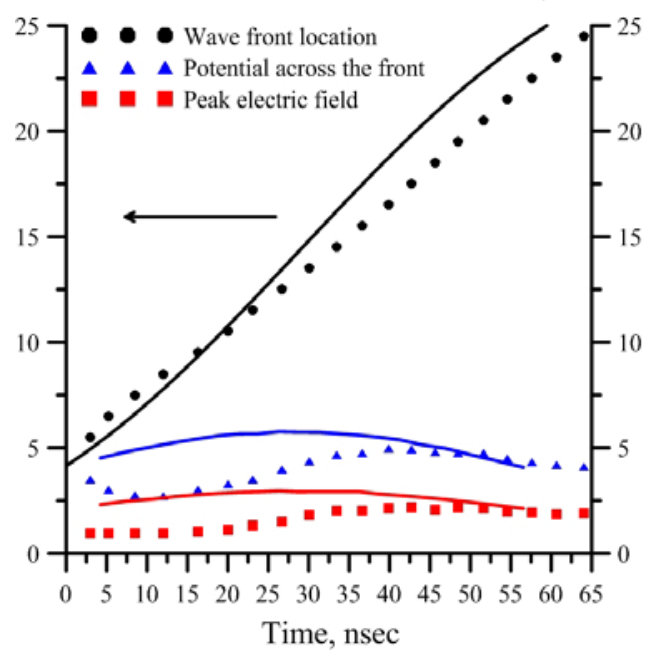

Figure 9. Comparison of experimental and predicted ionization wave front location vs. time, peak electric field, and potential across the wave front at different locations. Helium, $\mathrm{P}=20$ torr, $v=20 \mathrm{~Hz}, \mathrm{U}=500 \mathrm{~V}$, positive polarity wave. Numerical calculations using a quasi-onedimensional FIW discharge model.

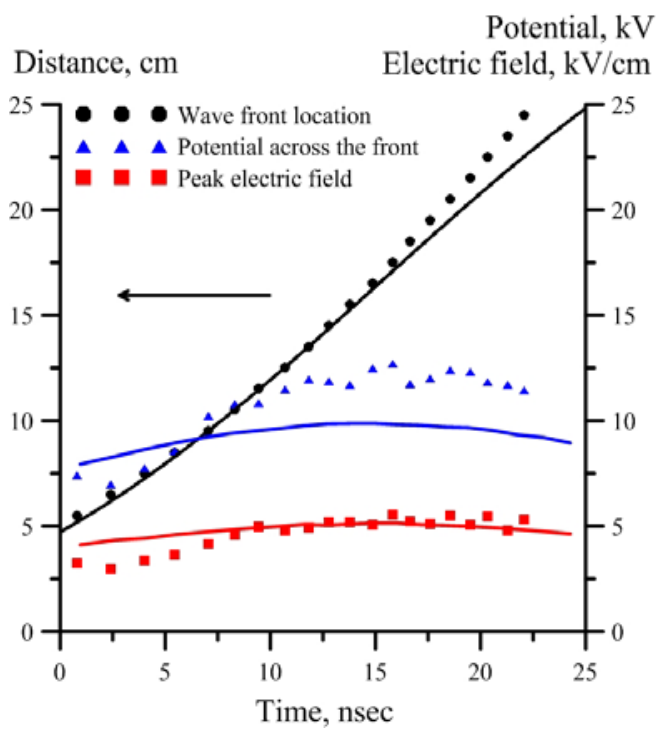

(a)

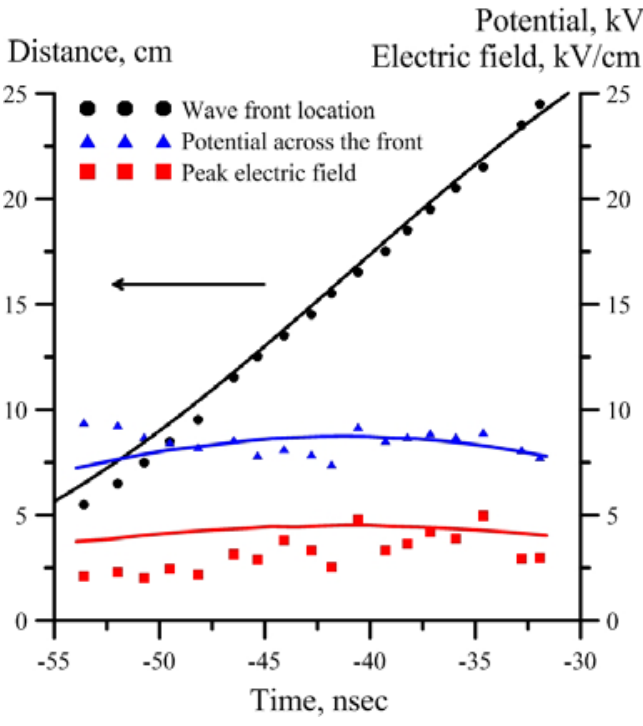

(b)

Figure 10. Comparison of experimental and predicted ionization wave front location vs. time, peak electric field, and potential across the wave front at different locations. Nitrogen, $\mathrm{P}=10$ torr, $v=20 \mathrm{~Hz}, \mathrm{U}=760 \mathrm{~V}$, (a) positive and (b) negative polarity waves. Numerical calculations using a quasi-one-dimensional FIW discharge model. 
(a)
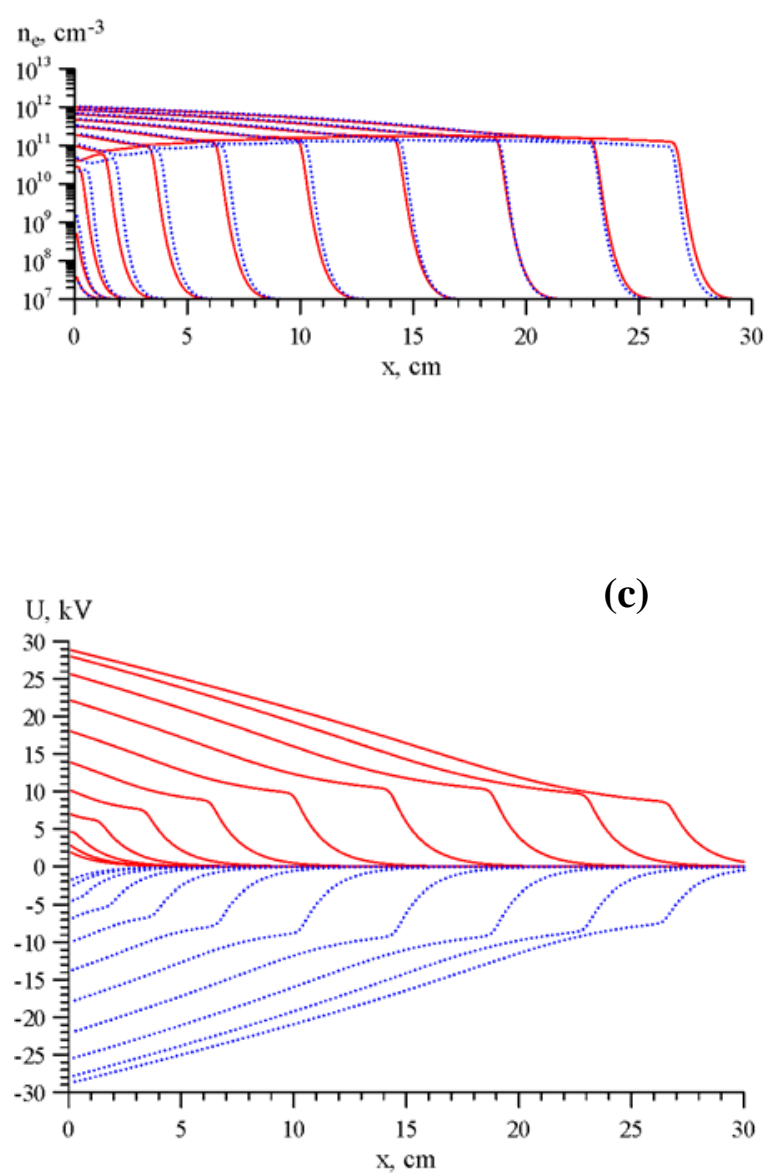

(b)

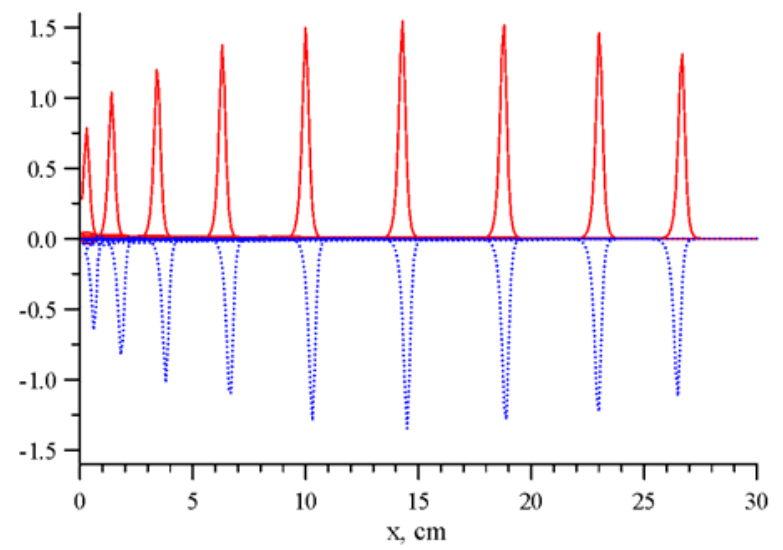

(d)

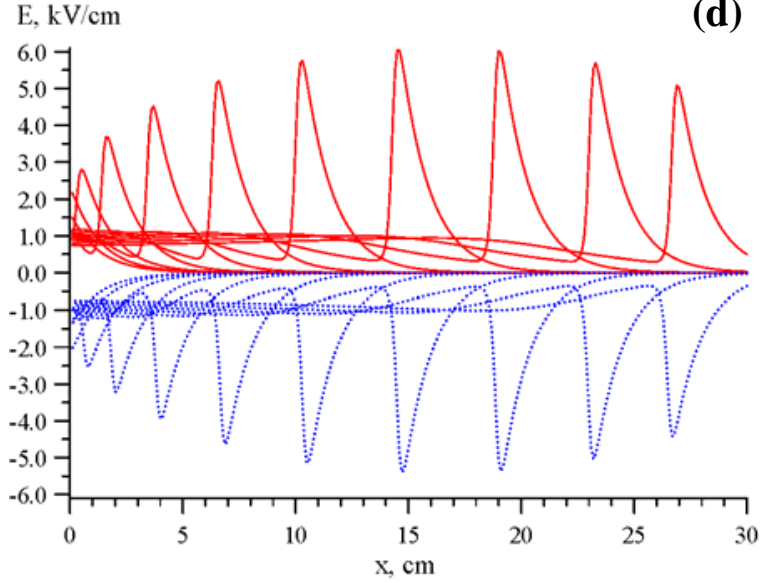

Figure 11. Distributions of (a) electron density, (b) space charge density, (c) potential, and (d) axial electric field in the FIW discharge in nitrogen at the conditions of Fig. 10. Solid curves, positive polarity wave; dashed curves, negative polarity wave. All distributions are plotted for multiple moments of time $5 \mathrm{nsec}$ apart. Numerical calculations using a quasi-one-dimensional FIW discharge model. 
Axial electric field, $\mathrm{V} / \mathrm{cm}$

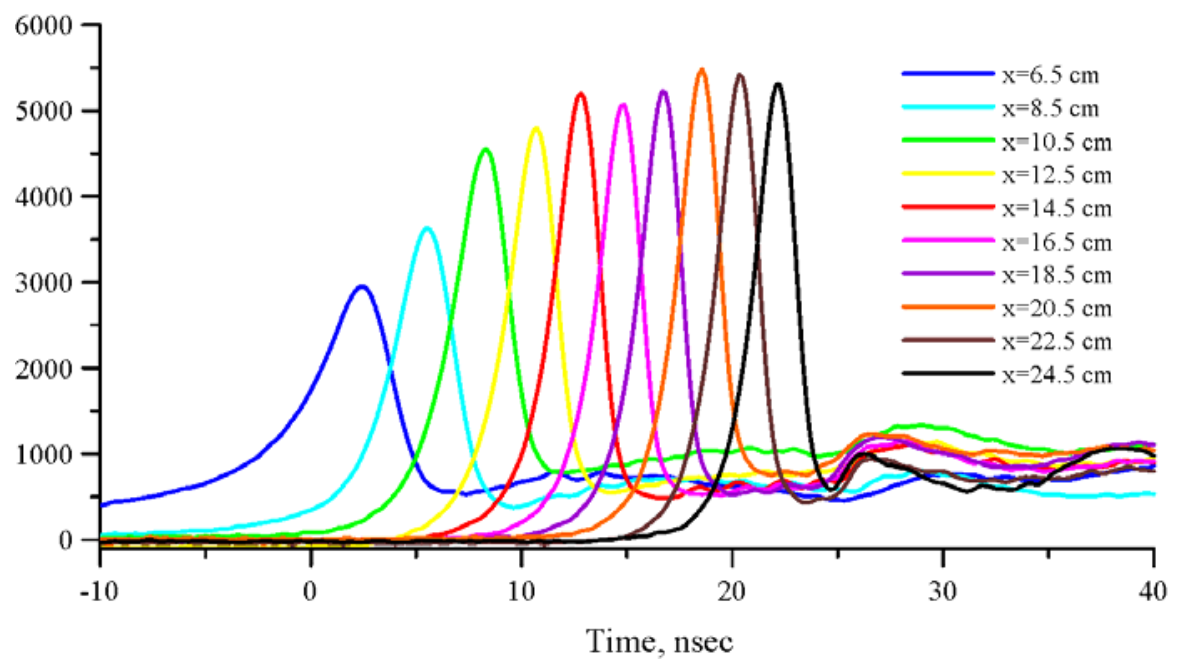

(a)

Axial elecric field, $\mathrm{V} / \mathrm{cm}$

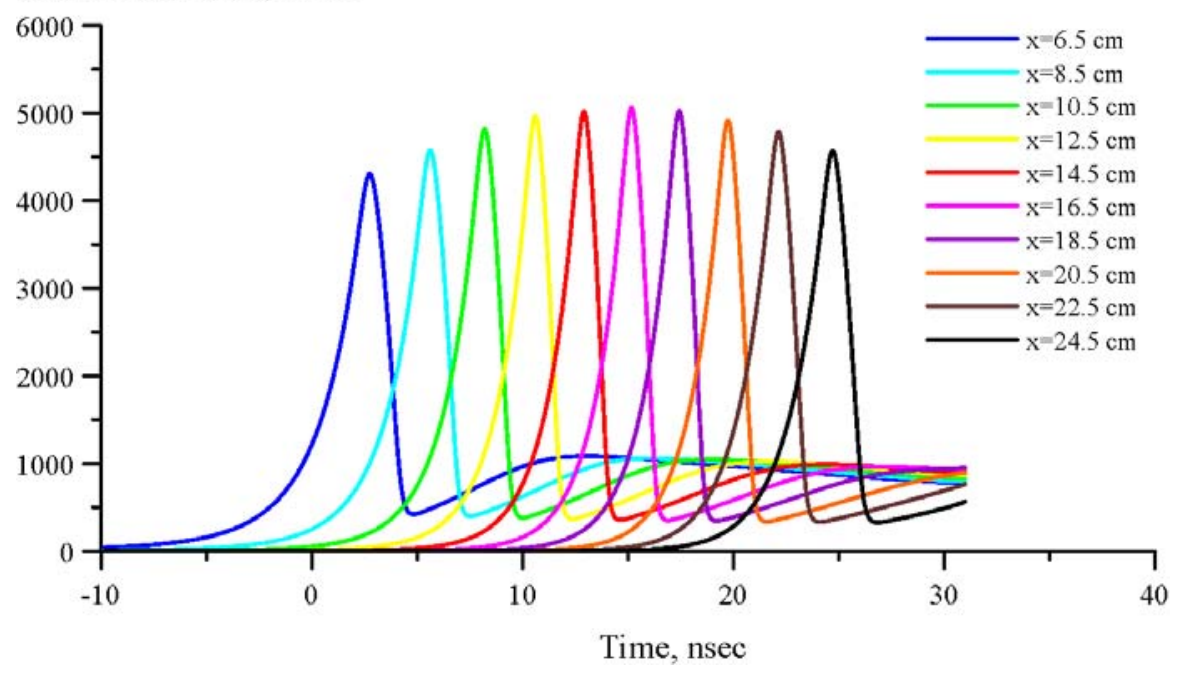

(b)

Figure 12. Comparison of (a) experimental and (b) predicted time-resolved axial electric field at different distances from the high-voltage electrode at the conditions of Fig. 10(a) (positive polarity wave). Numerical calculations using a quasi-one-dimensional FIW discharge model. 

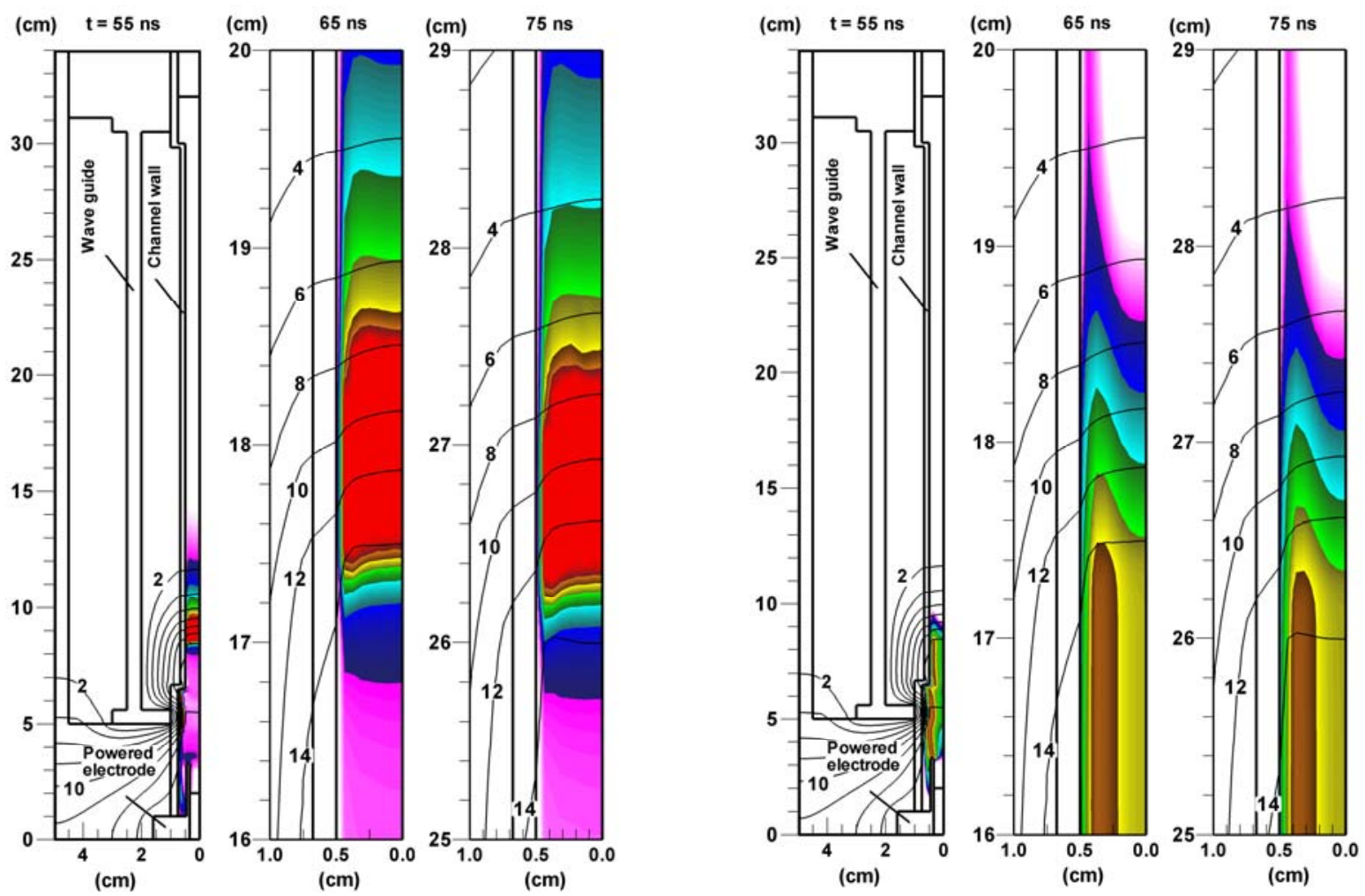

(a)

(b)

Figure 13. 2-dimensional FIW simulation of a positive polarity wave in nitrogen, at 10 Torr and U=760V. (a) electron temperature (flood contours) and electric potential (lines); (b) electron density (flood contours) and electric potential (lines), shown at $\mathrm{t}=55,65$ and $75 \mathrm{~ns}$. The spacing of the potential contour lines is $2 \mathrm{kV}$. Peak electron temperature $15 \mathrm{eV}$, peak electron density approximately $10^{12} \mathrm{~cm}^{-3}$. The wave speed is approximately $0.92 \mathrm{~cm} / \mathrm{nsec}$. 


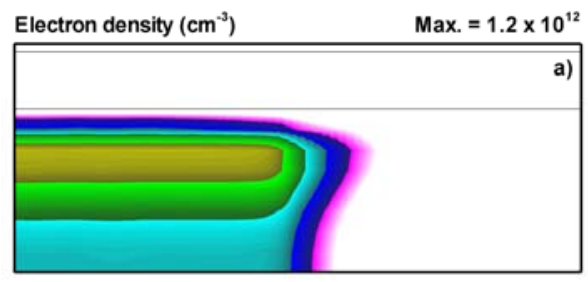

Axial electric field $\left(\mathrm{V}-\mathrm{cm}^{-1}\right) \quad 6.9 \times 10^{3}$
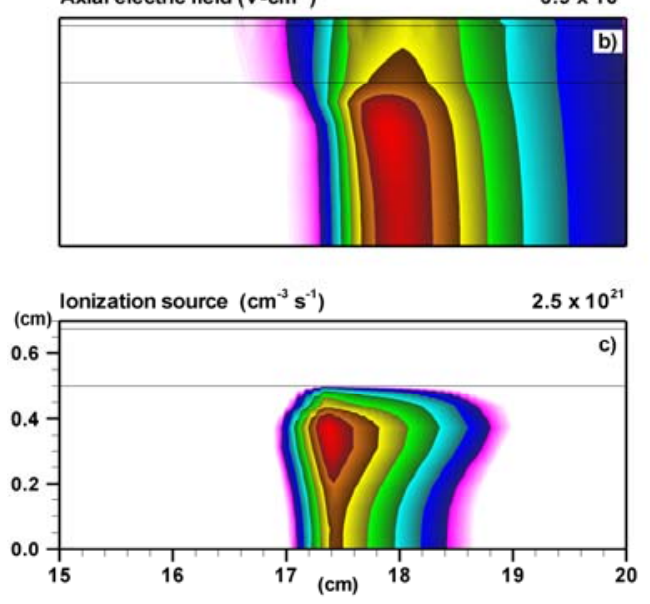

MIN MAX

Figure 14. Contours of a) electron density, b) axial electric field and c) ionization source rate, illustrating the structure of the ionization front at the conditions of Fig. 13, at $\mathrm{t}=65$ nsec.

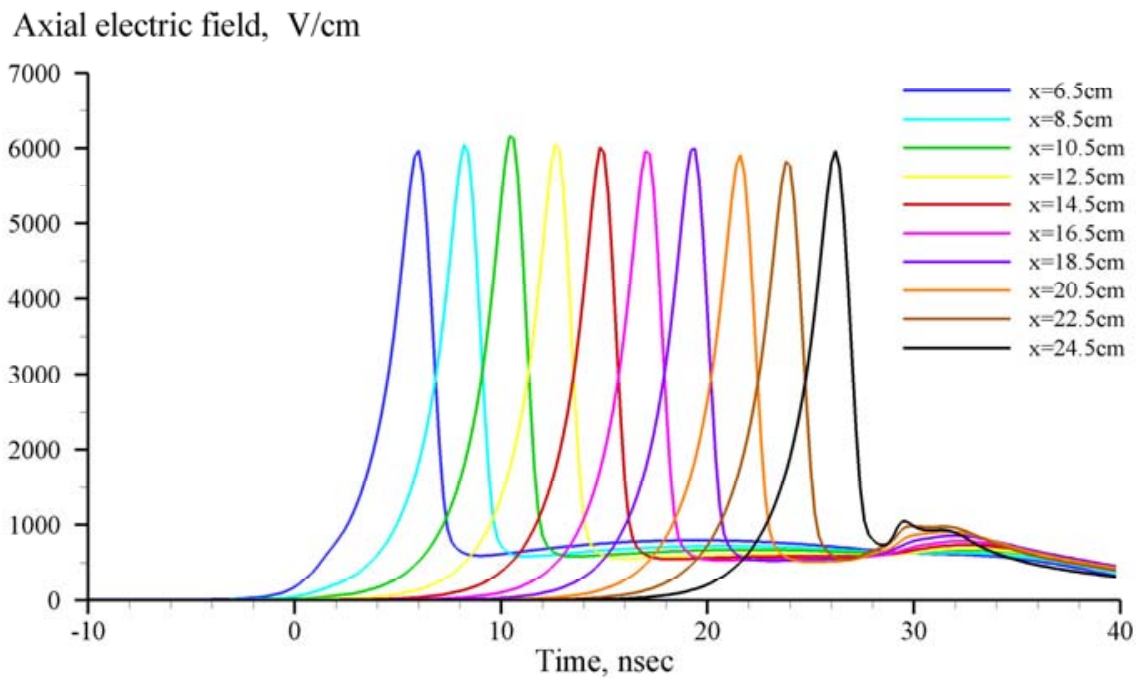

Figure 15. Time history of the electric field on the channel centerline predicted by the twodimensional model, for several axial locations of capacitive probes, at the conditions of Fig. 13. 


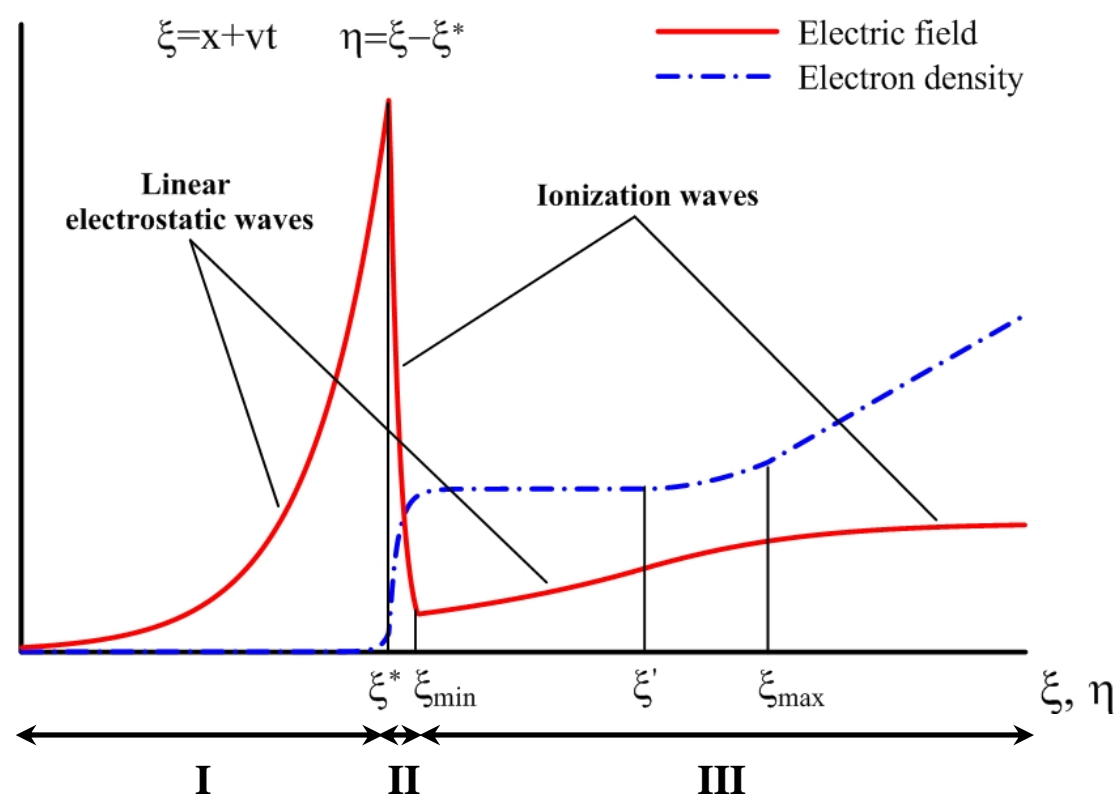

Figure 16. Schematic of a quasi-one-dimensional ionization wave structure 
Electron density, $\mathrm{cm}^{-3}$

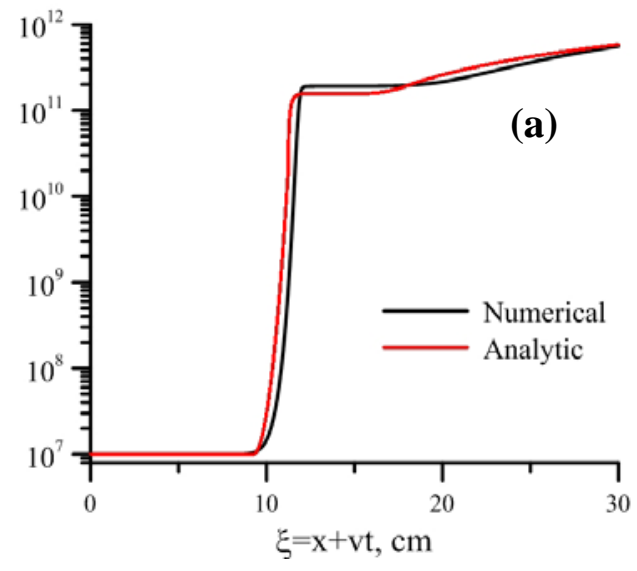

Potential, V

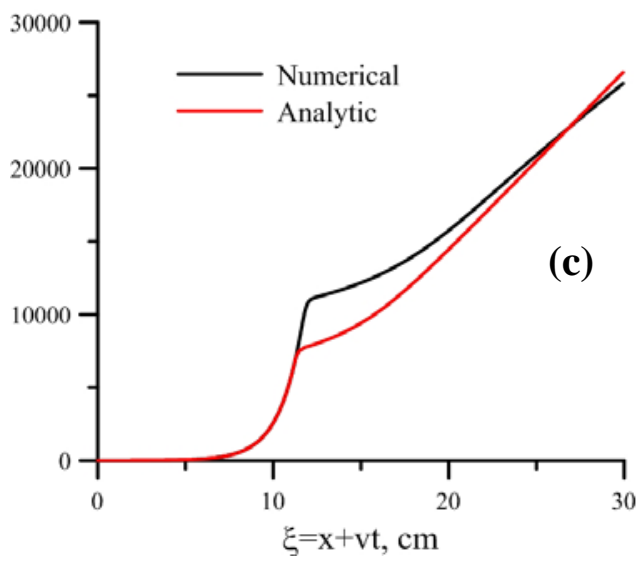

Space charge, $\mathrm{nC} / \mathrm{cm}^{3}$

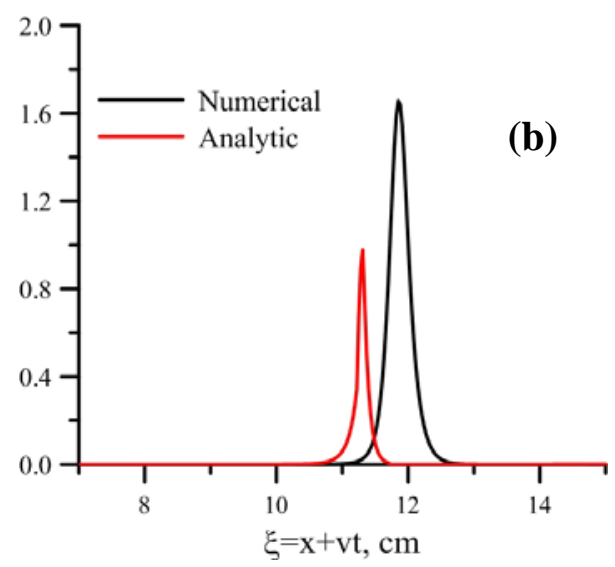

Axial electric field, $\mathrm{V} / \mathrm{cm}$

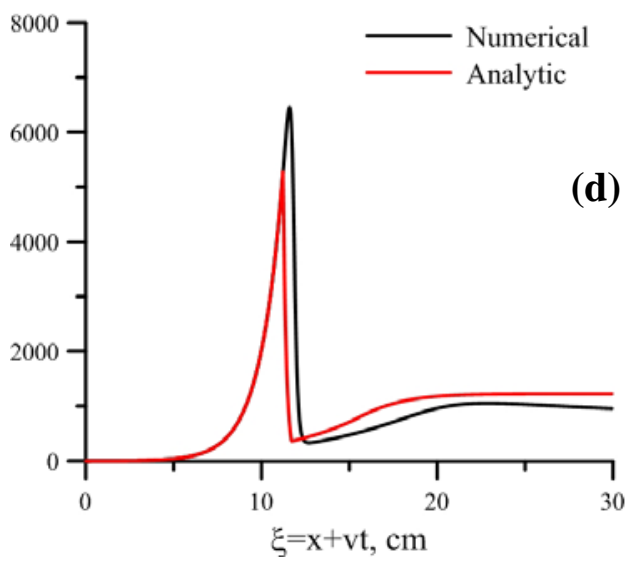

Figure 17. Comparison of numerical solution and self-similar analytic solution for (a) electron density, (b) space charge density, (c) potential, and (d) axial electric field in the wave front in nitrogen, at $\mathrm{P}=10$ torr and $V=1 \mathrm{~cm} / \mathrm{nsec}$ (positive polarity wave), i.e. close to the conditions of Fig. 10(a). 
Peak electric field $\left(\mathrm{E}^{*}\right)$

Residual electric field $\left(\mathrm{E}_{\max }\right), \mathrm{V} / \mathrm{cm}$

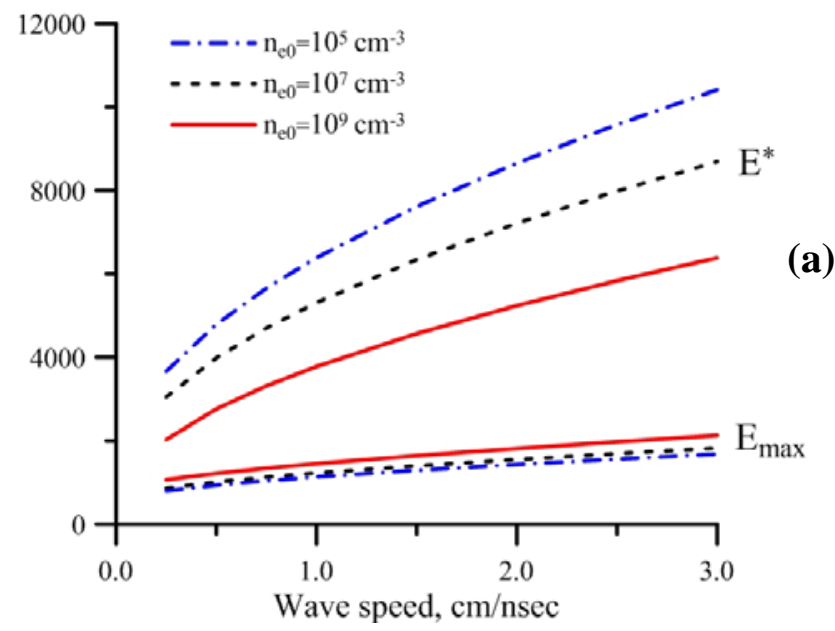

$\mathrm{dU} / \mathrm{dt}, \mathrm{kV} / \mathrm{nsec}$

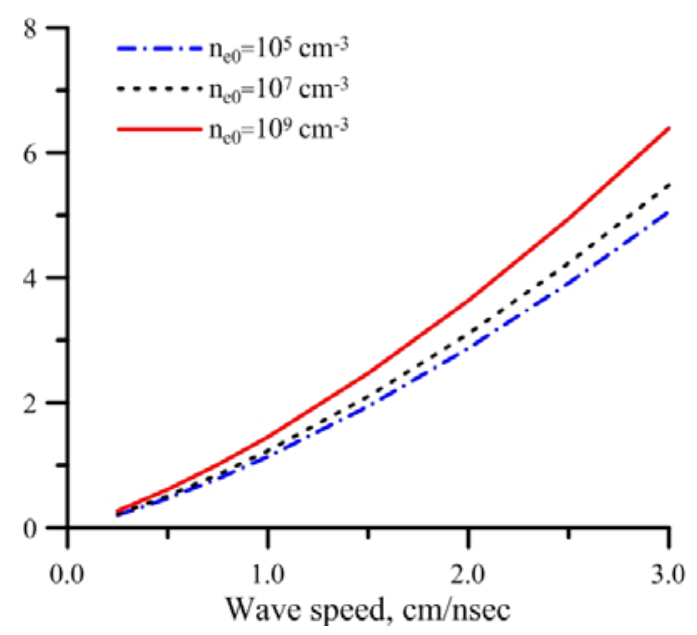

Electron density after primary wave, $\mathrm{cm}^{-3}$

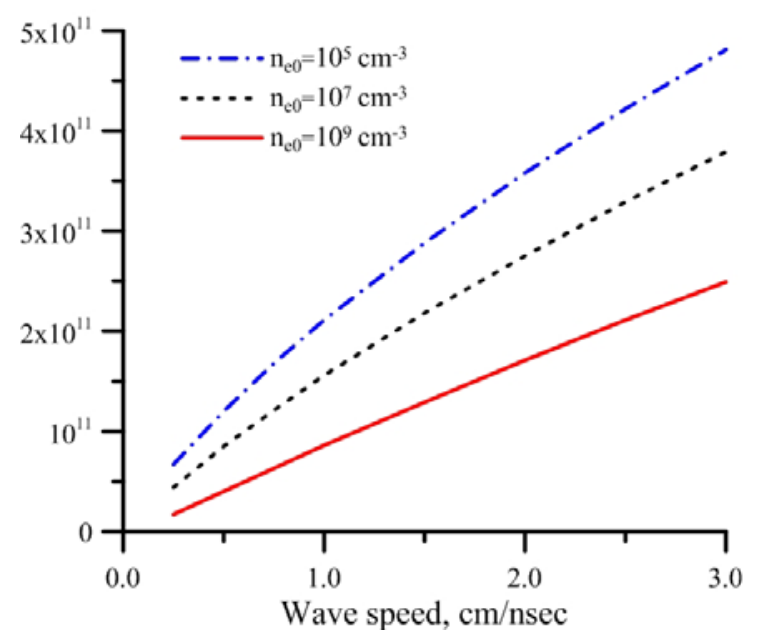

(b)

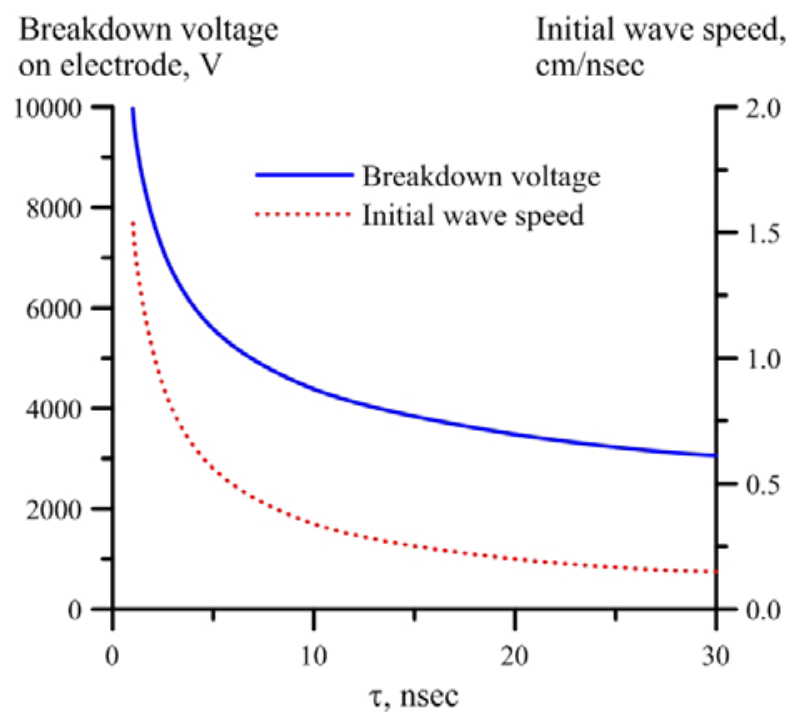

Figure 18. Summary of ionization wave parameters in nitrogen at $\mathrm{P}=10$ torr, predicted by the analytic model: (a) peak electric field and residual electric field vs. wave speed; (b) electron density achieved after the primary ionization wave vs. wave speed; (c) voltage rise rate on high-voltage electrode to maintain constant wave speed; (d) breakdown voltage near the electrode and initial wave speed for a Gaussian voltage pulse vs. pulse width. 